Revista Eletrônica de Direito Processual - REDP.

Rio de Janeiro. Ano 12. Volume 19. Número 2. Maio a Agosto de 2018

Periódico Quadrimestral da Pós-Graduação Stricto Sensu em Direito Processual da UERJ

Patrono: José Carlos Barbosa Moreira (in mem.). ISSN 1982-7636. pp. 84-117

www.redp.uerj.br

\title{
INTRODUZIONE AL C.D ACTIVE CASE MANAGEMENT NELLA PROCEDURA CIVILE: PREMESSE E SFIDE ${ }^{1}$
}

\section{INTRODUCTION TO ACTIVE CASE MANAGEMENT IN THE CIVIL PROCEDURE LAW: PREMISES AND CHALLENGES}

Elisângela Simon Caureo

Juíza Federal. Doutoranda em Processo Civil na Università degli Studi di Roma TRE, Italia, e também vinculada à Universidade Federal do Rio Grande do Sul, em regime de cotutela. Mestre em Direito pela UFRGS. Porto Alegre/RS. E-mail: elisangelacaureo@yahoo.com.br

RIASSUNTO: La moderna procedura civile e la giustizia manageriale sono concentrati sull'idea di una procedura isonomica, giusta e che abbia una ragionevole durata. I temi dell'efficienza, del controllo del giudice, della elasticità del rito (il processo taylor-made $\mathrm{o}$ prêt-à-porter) e della proporzionalità tra costi e risultati ricevono particolare enfasi. Questo testo intende indicare gli elementi principali dell'active case management inglese, mediante l'esame delle sue principale strutture, in particolare alcuni aspetti della Riforma Woolf. Inoltre, obiettivo del presente lavoro è esaminare il ruolo della fase preparatoria nella procedura civile e la possibile influenza del case management in Italia.

PAROLE-CHIAVE: Procedura Civile. Case management. Sommarizzazione. Costi del processo. Discovery. Disclosure. Tendenze della procedura civile.

ABSTRACT: Modern civil procedure and managerial justice are concentrated in the idea of an equal, fair and reasonably fast procedure. Much emphasis is placed upon themes like efficiency, judicial control, adaptability of procedures (taylor-made or prêt-à-porter), proportionality of costs and results. This paper commences by reflecting upon some aspects of case management and some tendencies in Procedural Law. Thereafter it will reflect upon some aspects of Lord Woolf's reforms. It will also analyse the role of the

\footnotetext{
${ }^{1}$ Artigo recebido em 15/10/2017 e aprovado em 25/06/2018.
} 
Revista Eletrônica de Direito Processual - REDP.

Rio de Janeiro. Ano 12. Volume 19. Número 2. Maio a Agosto de 2018

Periódico Quadrimestral da Pós-Graduação Stricto Sensu em Direito Processual da UERJ

Patrono: José Carlos Barbosa Moreira (in mem.). ISSN 1982-7636. pp. 84-117

www.redp.uerj.br

preparatory phase in the civil procedure and the possible influence of case management in Italy.

KEY WORDS: Case management. Litigation costs. Procedural law. Discovery. Disclosure. Procedural Law tendencies.

\section{Introduzione}

Antoine Garapon sostiene che la razionalità neoliberale ha stabilito le sue regole anche nell'ambito giudiziale. Da questo punto di vista, oggi ci troviamo davanti ad un nuovo periodo, quello che lui chiama "stato manageriale della governabilità", fase successiva al periodo rituale e disciplinare ${ }^{2}$.

La giustizia manageriale, affermò Garapon ${ }^{3}$, è la giustizia della tabellarizzazione di certe prestazioni, dell'adozione di protocolli destinati a specifici aspetti dell'atto giuridico, di formalizzazione e controllo riguardo ad atti antecedentemente realizzati in modo artigianale. È la risposta concettualmente più adatta ad una società intensamente bisognosa di normatizzazione, di efficienza normativa e di efficienza del servizio giudiziario.

È chiaro che nella moderna procedura civile l'obiettivo principale è quello di garantire alla società l'idoneità del sistema di produrre decisioni, come risultato di un procedimento equo, in conformità con le garanzie costituzionale della procedura civile e che abbia esito in breve tempo. In tale direzione, si conferma l'interesse verso una procedura efficiente, armoniosa, controllata, qualificata a soddisfare le necessità di un mondo moderno dominato dalla razionalità di mercato, che abbia la stessa celerità richiesta dalla dinamica contemporanea e che i suoi costi siano principalmente giusti e ragionevoli.

In tali circostanze, dal punto di vista del diritto continentale europeo, ottenere il grado di efficienza desiderato rappresenta una sfida importante per la procedura civile.

\footnotetext{
${ }^{2}$ In GARAPON A., Lo Stato Minimo. Milano: Raffaello Cortina Editore, 2012, p. 11: Secondo Garapon, il periodo rituale fu quello spazio di una Giustizia il cui traguardo era riportare la pace sociale, cioè a dire garantire il rispetto alla legge. Successivamente, nel periodo della disciplina la meta era quella di reinserire, rieducare e stabilire misure di assistenza sociale. Il terzo e attuale modello è invece rappresentato dalla giustizia manageriale.

${ }^{3}$ IVI, p. 40.
} 
Revista Eletrônica de Direito Processual - REDP.

Rio de Janeiro. Ano 12. Volume 19. Número 2. Maio a Agosto de 2018

Periódico Quadrimestral da Pós-Graduação Stricto Sensu em Direito Processual da UERJ

Patrono: José Carlos Barbosa Moreira (in mem.). ISSN 1982-7636. pp. 84-117

www.redp.uerj.br

Anche in questa circostanza, sorge e acquista riconoscimento e poteri un modello

di gestione del processo che dà ai giudici l'opportunità di operare su livelli che erano ancora vietati: 1' "active case management".

Questo testo mostra gli elementi principali dell'active case management inglese, attraverso l'esame delle sue principali strutture, con l'approccio di alcune categorie del diritto nord americano. Tale motivazione si origina da un periodo della procedura brasiliana che si avvicina ad alcune strutture di common Law, nonché della processualistica italiana in cui si cerca di rendere flessibile il proprio rito processuale.

"Mettere il processo nelle mani del giudice", è questo il contributo che secondo Neil Andrews è stato apportato dal sistema di case management, e non solo. Infatti, ha anche l'obiettivo di assicurare che il processo segua i binari giusti e che non venga manipolato dalle parti del processo: "The CPR system (1998) places management of cases in the hands of judges. Before 1999, too many cases had been left to drift without official direction. These disputes had become the (lucrative) play-thing of rivel teams of lawyers ",

Il tema da affrontare è quindi il ruolo del giudice come manager del processo, il quale è dotato di ampi poteri nella procedura civile, permettendo loro di conformare il rito processuale all'oggetto della lite; in tal modo, riescono ad ottenere dal sistema la massima efficacia possibile.

In tal senso, l'obiettivo principale è quello di elaborare concetti, istituti e caratteristiche proprie del case management e dei poteri di case manager, identificando $\mathrm{i}$ vantaggi pratici di questo approccio della procedura civile moderna.

\section{Case management: il controllo del giudice}

"Who is to impose, encourage or achieve efficiency at such an early stage of a case? My suggestion is that only a judge may do so",5. Le suggestive parole di Marco Bonci indicano chi è il protagonista della procedura civile nel case management.

Il ruolo del giudice inizia a mutare già a partire dalla metà del secolo scorso, attenuando la giustapposizione fra sistemi di common Law e di civil Law ${ }^{6}$. Ansanelli,

\footnotetext{
${ }^{4}$ ANDREWS N., The Three Paths of Justice. Dordrecht: Springer, 2012, p. 23.

${ }^{5}$ BONCI M., Active Case Management - English Reception and Italian Rejection, RePro, v. 38, n. 219, p. 225-237, maggio 2013, p. 226.

${ }^{6}$ DONDI A.-ANSANELLI V.-COMOGLIO P., Processi Civili in Evoluzione, Milano: Giuffrè Editore, 2015,
} 
Revista Eletrônica de Direito Processual - REDP.

Rio de Janeiro. Ano 12. Volume 19. Número 2. Maio a Agosto de 2018

Periódico Quadrimestral da Pós-Graduação Stricto Sensu em Direito Processual da UERJ

Patrono: José Carlos Barbosa Moreira (in mem.). ISSN 1982-7636. pp. 84-117

www.redp.uerj.br

Comoglio e Dondi sostengono che il profilo attivo del giudice non è una novità nelle

diverse culture giuridiche, allorché viene menzionato il profilo scelto dal legislatore italiano del 1940 rispetto a tale figura, ma che non si è imposto, come tale, nel quotidiano della pratica giurisdizionale ${ }^{7}$.

Tuttavia, è necessario menzionare, al fine di non trascurare la verità storica, che la preoccupazione riguardo al ruolo attivo del giudice è già presente nelle idee di Franz Klein concretizzate nella Z.P.O austriaca del 1895, come rileva autorevolmente Carratta ${ }^{8}$ richiamando il pensiero di Chiovenda:

(...) coll'idea che l'amministrazione della giustizia sia una funzione della sovranità, si viene formando lentamente la convinzione che il giudice come organo dello Stato non debba assistere passivamente alla lite, per pronunciare alla fine una sentenza, come l'automa eccitato dal peso della moneta cadente emette un dolce o un biglietto d'ingresso: ma debba partecipare alla lite come forza viva ed attiva.

Già nel contesto di common Law, gli ordinamenti degli Stati Uniti e dell'Inghilterra hanno progressivamente ristrutturato i poteri del giudice nel processo civile, passando da un modello strutturato su capisaldi adversariali - che non gli consentivano di esercitare un'attività manageriale attiva ma di mero controllore del procedimento - ad un ruolo attivo del giudice, capace di far fronte ai problemi più complessi del percorso giudiziale (durata, liti complesse, collettive, costi) ${ }^{9}$. Anche gli ordinamenti francese e spagnolo seguono questa direzione rispetto all'evoluzione della funzione del giudice ${ }^{10}$.

In Inghilterra, le Civil Procedure Rules del 1998 hanno cambiato radicalmente la procedura civile. Queste norme, infatti, modificarono la cultura originaria della English civil litigation $^{11}$, gli Judicature Acts ${ }^{12}$, e favorirono un ruolo di maggior coinvolgimento del giudice nell'avanzamento della lite e nel suo svolgimento formale.

p. 45.

${ }^{7}$ IVI, p. 45.

${ }^{8}$ Apud CARRATTA A., Funzione sociale e processo civile fra XX e XXI secolo, Disponibile su: http://romatrepress.uniroma3.it/ojs/index.php/funzione/article/view/506/503. Accesso il: 17 ottobre 2017, p. 118 (Chiovenda, Le riforme processuali, cit. nt. 114, p. 385).

${ }^{9}$ DONDI A.-ANSANELLI V.-COMOGLIO P., Processi Civili in Evoluzione. Milano: Giuffrè Editore, 2015, p. 47.

${ }^{10}$ IVI, p. 47.

${ }^{11}$ FICCARELLI B., Fase Preparatória del Processo civile e case management giudiziale, Napoli: Edizione Scientifiche Italiane, 2011, p. 93. 
Revista Eletrônica de Direito Processual - REDP.

Rio de Janeiro. Ano 12. Volume 19. Número 2. Maio a Agosto de 2018

Periódico Quadrimestral da Pós-Graduação Stricto Sensu em Direito Processual da UERJ

Patrono: José Carlos Barbosa Moreira (in mem.). ISSN 1982-7636. pp. 84-117

www.redp.uerj.br

In effetti, è un'affermazione presente nella dottrina, la quale afferma che la storia

della procedura inglese può essere divisa in tre parti: (a) il primo periodo, antecedente alle riforme del 1875; (2) il secondo periodo che racchiude le riforme del 1875 e il codice del 1999; (3) il terzo periodo successivo al $1999^{13}$.

Il periodo che precede le riforme presenta delle caratteristiche essenzialmente adversariali ed è conosciuto come lo spazio del principio "party control". Con le parole di Lord Woolf: "la condotta, il ritmo la durata della controversia sono lasciate pressoché totalmente alle parti" ${ }^{\prime 14}$. Inoltre spiega:

(...) senza un effettivo controllo da parte del giudice...il processo adversary può incoraggiare una cultura adversary e degenerare in un ambiente nel quale il processo è troppo spesso visto come un campo di battaglia dove non vige alcuna regola. In questo ambiente questioni di costo, tempo, compromesso e di giustizia non possono che avere bassa priorità ${ }^{15}$.

Lord Woolf ha compreso che per superare il problema dei costi giudiziari in Inghilterra, oggetto di indagine nelle prossime pagine di questo testo, si necessitava di un approccio di controllo sui riti procedurali, riducendo l'abuso all'interno del processo: "The existence of different modes of processing disputes and the ever watchful eye of the judiciary will aim to ensure that the course of litigation adopted in any given case will be in reasonable proportion to the complexity and importance of the dispute"16.

Di conseguenza, accanto al controllo legale del rito processuale, Lord Woolf ha percepito la necessità di una seconda forma, il controllo del giudice, che da questo momento in poi assume il ruolo di organizzatore del processo.

Va inoltre osservato che la English Commercial Court $^{17}$ possedeva già una tradizione di trattazione riguardo alle domande cross-border e alle domande di alto valore finanziario. Pertanto hanno sviluppato poteri manageriali sin dall'inizio di ogni domanda

12 ZUCKERMAN A.A.S., Lord Woolf's Access To Justice: Plus Ça Change, in (1996) 59 Modern Law Review 77: "The grotesque procedures before the Judicature Acts and their extravagant delays and excessive costs were shown up by Dickens in Bleak House, written in 1852”, p. 7.

${ }^{13}$ DONZELLI R., La fase preliminare del nuovo processo civile inglese e l'attività di "case management" giudiziale, in Davanti al giudice. Studi sul processo societario, a cura di L. Lanfranchi e A. Carratta, 2005, Torino, p.11.

${ }^{14}$ IVI, p. 12.

15 IBIDEM.

${ }^{16}$ ZUCKERMAN A. A. S., Lord Woolf's Access to Justice: Plus Ça Change, in 59 Modern Law Review 77, 1996, p. 8.

${ }^{17} \mathrm{http}$ ///ce-file.uk/onewebmedia/admiralty-and-commercial-courts-guide.pdf: Le Commercial Court inglesi hanno un loro proprio codice procedurale. Nel 2011 la Commercial Court Guide ha inoltre redatto il manuale chiamato: "Key features of case management", aggiornato a marzo 2016. 
Revista Eletrônica de Direito Processual - REDP.

Rio de Janeiro. Ano 12. Volume 19. Número 2. Maio a Agosto de 2018

Periódico Quadrimestral da Pós-Graduação Stricto Sensu em Direito Processual da UERJ

Patrono: José Carlos Barbosa Moreira (in mem.). ISSN 1982-7636. pp. 84-117

www.redp.uerj.br

processuale $^{18}$.

Il modello di "case management" nacque, quindi, in generale, con l'obiettivo di rendere il processo idoneo a promuovere una giustizia più effettiva e accessibile e come soluzione a situazioni di esaurimento dei sistemi. In tale modello, effettività e accessibilità hanno stretta relazione con strumenti di riduzione dei costi e dei tempi del processo. Secondo Ficcarelli, uno degli effetti più visibili della riforma, introdotta dal modello di case management inglese, è la riduzione della durata dei processi: "Cases that took five years or more to come to trial - scrive Turner - are now resolved in fifteen or eighteen month" $"$.

Nella prima parte delle Civil Procedure Rules del 1998, con il titolo "Overriding Objective", vi sono i principi e gli obiettivi che guidano l'attività processuale ${ }^{20}$. Ed è in

${ }^{18}$ BONCI M., Active Case Management - English Reception and Italian Rejection. Revista de Processo: RePro, v. 38, n. 219, p. 225-237, maio 2013. p. 230: "Here one can contrast the sophisticated tradition of the English Commercial Court. This is a specialist branch of the High Court, based in London, and dealing with many cross-border and high value disputes. For several decades the Commercial Court judges (who are known to be pioneers in procedural matters) have made intelligent, resourceful, and enthusiastic use of managerial powers from the early stage of such dispute. These judges hear all pre-trial applications. They make case management hearings. This is stated to be for this purpose: "to achieve definition of real matters in issue as early as possible with as few technicalities and as few interlocutory [that is, pre-trial] proceedings as were consistent with an orderly trial".

${ }^{19}$ FICCARELLI B., Fase Preparatória del Processo civile e case management giudiziale. Napoli: Edizione Scientifiche Italiane, 2011, p. 135: "Più dettagliati e quindi anche più probanti I dati tratti da Zander dale Judicial Statistics annuali, dai quail risulta che la durata media dei giudizi di fronte alle county courts che nel 1990 era di 81 settimane e nel 1998 di 85 settimane, nel 2005 si è ridotta a 52 settimane e che la durata media dei casi assegnati al fast track dal momento dell'assegnazione a tale modulo processuale e la data del trial si mantine nell'ambito delle 30 settimane volute dalle Civil Procedure Rules".

20 "The overriding objective

1.1(1) These Rules are a new procedural code with the overriding objective of enabling the court to deal with cases justly and at proportionate cost. (2) Dealing with a case justly and at proportionate cost includes, so far as is practicable -(a) ensuring that the parties are on an equal footing; (b) saving expense; (c) dealing with the case in ways which are proportionate -(i) to the amount of money involved;

(ii) to the importance of the case; (iii) to the complexity of the issues; and (iv) to the financial position of each party; (d) ensuring that it is dealt with expeditiously and fairly; (e) allotting to it an appropriate share of the court's resources, while taking into account the need to allot resources to other cases; and (f) enforcing compliance with rules, practice directions and orders. Application by the court of the overriding objective:1.2The court must seek to give effect to the overriding objective when it -(a) exercises any power given to it by the Rules; or (b) interprets any rule subject to rules 76.2, 79.2 and 80.2, 82.2 and 88.2.

Duty of the parties 1.3 The parties are required to help the court to further the overriding objective. Court's duty to manage cases 1.4 (1) The court must further the overriding objective by actively managing cases. (2) Active case management includes -

(a) encouraging the parties to co-operate with each other in the conduct of the proceedings; (b) identifying the issues at an early stage; (c) deciding promptly which issues need full investigation and trial and accordingly disposing summarily of the others; (d) deciding the order in which issues are to be resolved;

(e) encouraging the parties to use an alternative dispute resolution(GL)procedure if the court considers that appropriate and facilitating the use of such procedure; (f) helping the parties to settle the whole or part of the case; (g) fixing timetables or otherwise controlling the progress of the case; $(\mathrm{h})$ considering whether the 
Revista Eletrônica de Direito Processual - REDP.

Rio de Janeiro. Ano 12. Volume 19. Número 2. Maio a Agosto de 2018

Periódico Quadrimestral da Pós-Graduação Stricto Sensu em Direito Processual da UERJ

Patrono: José Carlos Barbosa Moreira (in mem.). ISSN 1982-7636. pp. 84-117

www.redp.uerj.br

queste regole che il legislatore inglese ha coniato i valori di questa nuova cultura processuale, marcata da parole come flessibilità ed elasticità e sotto l'influsso concettuale della proporzionalità ${ }^{21}$.

Il modello processuale di active case management è caratterizzato da un'attuazione cooperativa delle parti e dagli ampi poteri conferiti al giudice nell'identificare le questioni controverse, le questioni complesse che esigono attività probatoria più completa, le questioni che possono essere decise sommariamente e la risoluzione alternativa della controversia ${ }^{22}$.

Altra novità inedita nei sistemi processuali, data la sua ampiezza, è che al giudice del case management è conferito il potere di scegliere il procedimento (track) in base alle caratteristiche individuali di ciascuna causa civile, personalizzando il rito secondo una valutazione di economia processuale ed efficacia, senza pregiudizio del diritto di difesa e della giustizia della decisione.

Il concetto si basa sull'idea che una relativa assenza di predeterminazione può significare una accentuata crescita di efficienza rituale. Tuttavia, secondo tale ipotesi, il managerial judge non opera solo riguardo al rito, in quanto anche la produzione della prova riceve i suoi influssi.

Per una panoramica più precisa, si ritiene necessario segnalare i principali passaggi della procedura inglese, che può essere divisa in sei tappe: (1) pre-action; (2) commencement and pleadings; (3) disclosure: party preparation of factual evidence, expert evidence, and exchange of documents between the parties (prima chiamata discovery); (4) trial; (5) appeal; (6) enforcement ${ }^{23}$.

Tenendo conto che le procedure civile di civil law non di rado hanno dimostrato assenza d'interesse riguardo al periodo che precede l'inizio formale del processo, in modo che non esiste, sia nella procedura civile brasiliana, sia nel processo civile italiano, un

likely benefits of taking a particular step justify the cost of taking it; (i) dealing with as many aspects of the case as it can on the same occasion; ( $j$ ) dealing with the case without the parties needing to attend at court;

(k) making use of technology; and (l) giving directions to ensure that the trial of a case proceeds quickly and efficiently".

${ }^{21}$ ANDREWS, op. cit., p. 10: “Tracks: The allocation of cases to different types of first instance court (county court or High Court) and to different systems of procedure (known as 'tracks') under the CPR (1998) is founded on the principle of jurisdictional proportionality: that litigation must be tailored to the size and nature of the dispute".

${ }^{22}$ FICCARELLI B., Fase preparatoria del processo civile e case management giudiziale, Napoli: Edizione Scientifiche Italiane, 2011, p. 135.

${ }^{23}$ ANDREWS N., The Three Paths of Justice. Dordrecht: Springer, p. 8. 
Revista Eletrônica de Direito Processual - REDP.

Rio de Janeiro. Ano 12. Volume 19. Número 2. Maio a Agosto de 2018

Periódico Quadrimestral da Pós-Graduação Stricto Sensu em Direito Processual da UERJ

Patrono: José Carlos Barbosa Moreira (in mem.). ISSN 1982-7636. pp. 84-117

www.redp.uerj.br

momento che si possa assimilare al fenomeno designato "disclosure", sicché bisogna descrivere le particolarità di questa fase e di quella della pre-action.

Nel processo inglese, è nelle fasi di pre-action (1) e disclosure (3) ${ }^{24}$ che occorrono i procedimenti di 'Pre-trial applications' e case management udienze (hearings).

Pre-action phase $e^{25}$ è il periodo che intercorre tra il sorgere del fondamento della domanda e l'inizio formale del procedimento. Il sistema del CPR (Civil Procedure Rules) ha stabilito una serie di pre-action protocols, ovvero di alcuni strumenti il cui obiettivo è promuovere accordi (concordato), evitando l'aumento dei costi del processo. La razionalità dei pre-actions protocols, è proprio quella di evitare il processo, nel senso che questo sia avvertito effettivamente solo come ultima ratio per la soluzione delle controversie.

Aspetti dei pre-actions protocols: a) self-executing and require the disputants to co-operate; b) le corti svolgono un controllo a posteriori; c) sono prescrizioni di obbligazioni che le parti devono soddisfare prima dell'inizio formale delle domande. "One of the aims of this system is that each side should know the strengths of his opponent's case $^{\text {"26. }}$. Un altro aspetto che merita di essere rilevato è quello dello scambio di informazioni, il quale gioca un ruolo importante nel preparare e stimolare il raggiungimento di un accordo. ${ }^{27}$

La preparazione della causa è collegata all'importanza che il sistema attribuisce al principio della Jurisdictional Proportionality, il quale, di fatto, stabilisce che il rito, deve corrispondere alla dimensione e alla natura della $\operatorname{lite}^{28}$. Una delle possibilità di

\footnotetext{
${ }^{24}$ IVI, p. 8.

${ }^{25}$ IVI, p. 8: "covers the period from when the relevant ground of complaint or contested issue arose until that matter produces formal civil proceedings".

${ }^{26}$ IVI, p. 9.

${ }^{27}$ IBIDEM: "It is also hoped that settlement will be promoted by efficient exchange of information. For example, a person who alleges that he was the victim of medical negligence can gain access to hospital or medical records under this system of pre-action protocols. If the dispute does proceed to a formal action, the court has power to sanction a person's failure to comply by making an appropriate costs order. Various rules or judicial powers regulate preservation of, or access to, potential evidence and information before formal commencement of proceedings".

28 ANDREWS, op. cit., p. 10: “Tracks: The allocation of cases to different types of first instance court (county court or High Court) and to different systems of procedure (known as 'tracks') under the CPR (1998) is founded on the principle of jurisdictional proportionality: that litigation must be tailored to the size and nature of the dispute. The idea of allocating cases to different types of court according to their value or importance was not an innovation of the CPR system. The dualism of county court a High Court first instance jurisdiction can be traced to the nineteenth century. Within the county court siystem, small claims procedure was introduced in the 1960s and 1970s. These matters, therefore, ante-date the Woolf Inquiry of 1994-1996. However, the CPR (1998) system does refine the notion of proportionate allocation (stopping
} 
Revista Eletrônica de Direito Processual - REDP.

Rio de Janeiro. Ano 12. Volume 19. Número 2. Maio a Agosto de 2018

Periódico Quadrimestral da Pós-Graduação Stricto Sensu em Direito Processual da UERJ

Patrono: José Carlos Barbosa Moreira (in mem.). ISSN 1982-7636. pp. 84-117

www.redp.uerj.br

applicazione della proporzionalità viene attuata mediante la presenza di tre riti procedurali:

Small claims jurisdiction; Fast-track e Multi-track.

Lo Small claims jurisdiction riguarda le domande che non eccedono $£ 10,000$ (o $£ 1,000$, per casi specifici); tali domande devono essere proposte dinanzi al giudice delle County Courts (prima istanza di giurisdizione); nella Fast-Track vi sono le domande che non eccedono $£ 25,000$; al Multi-Track, tutte le altre domande. È importante, inoltre, segnalare che le High Courts ricevono le domande che superano $£ 50,000$.

I criteri di allocazione delle domande sono:

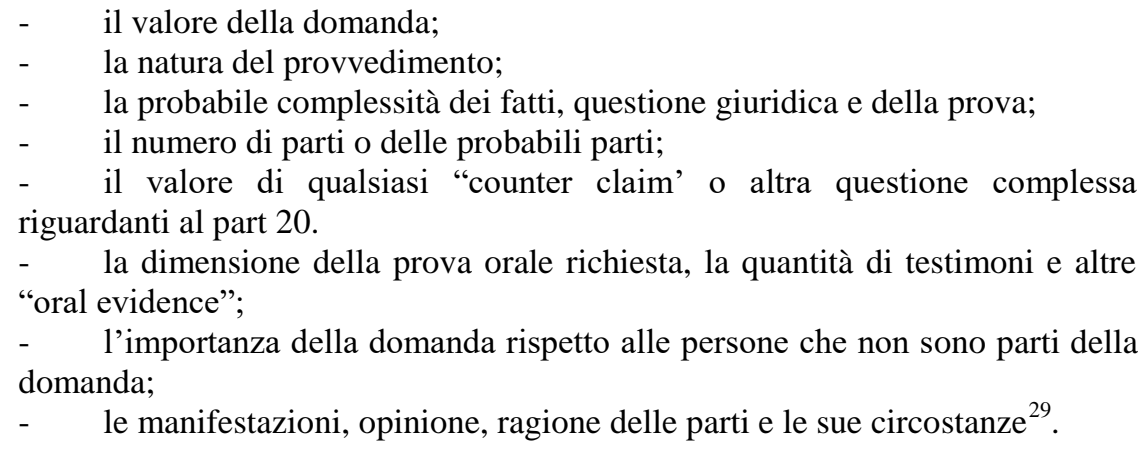

L'allocazione può essere comunque modificata dalla corte. La domanda, nel processo inglese, comincia quando la parte ha compilato le suddette claims form (Directions form), che sono nient'altro che formulari, in cui sono richieste le informazioni più importanti, per stabilire la corretta allocazione del rito.

La procedura inglese prevede ampi poteri ai giudici (Court). Essi devono garantire l'allocazione di risorse delle corti in maniera proporzionale, non solo rispetto alle corti, ma anche rispetto alle parti, ovvero devono controllare il rapporto di costo del processo e il beneficio che si aspetta di raggiungere. Determinare il "track", il rito adeguato, in questo caso, significa migliorare il rito, ridurre i costi ed accelerare il processo.

\footnotetext{
short of amalgamating the county court and High Court system into a unified first instance court). There are now three tracks (that is, types of first instance procedure to which a case can be allocated): the small claims jurisdiction; the fast-track; and the multi-track. High court litigation is concerned only with the multi-track. The county courts, which are the inferior first instance jurisdiction, are concerned with all three tracks. At the time of writing (April 2011), the small claims system concerns actions not exceeding $£ 5,000$ (or $£ 1,000$ in the case of personal injury claims and housing repair caims by resident tenants). (...) Above the small claims jurisdiction is the second tier of first instance adjudication, the 'fast track', dealing with claims not exceeding $£ 25,000$. At the top of the first instance system, the third tier, is the verstiel 'multi-track', dealing with all other actions. The High Court tends not to deal with matters less than $£ 50,000$ ".
}

${ }^{29}$ ANDREWS, op. cit., p. 11. 
Revista Eletrônica de Direito Processual - REDP.

Rio de Janeiro. Ano 12. Volume 19. Número 2. Maio a Agosto de 2018

Periódico Quadrimestral da Pós-Graduação Stricto Sensu em Direito Processual da UERJ

Patrono: José Carlos Barbosa Moreira (in mem.). ISSN 1982-7636. pp. 84-117

www.redp.uerj.br

Secondo l'autore Andrews $^{30}$, i giudici possiedono, nei termini del CPR, la specifica responsabilità di:

(a) "Co-operation and settlement: encouraging co-operation between the parties; helping parties to settle all or part of the case; encouraging alternative dispute resolution; if necessary, staying the action to enable such extra-curial negotiations or discussion to be pursue;",

(b) Determinare le questioni rilevanti e le priorità: vale a dire identificare la/e questione/i controversa/e; stabilire l'ordine di importanza e il rapporto di pregiudizialità tra di loro; stabilire il rito, se più ampio e complesso oppure sommario;

(c) “making summary decision", cioè a dire prendere decisioni sommarie: decidere sull'assunzione dei summary hearings (CPF Part 24); sull'estinzione del processo per vizi o manifesta infondatezza; pronunciare decisioni provvisorie; impulsare il processo attraverso il calendario del processo e tutti gli altri aspetti del tramite procedurale che possono influenzare il percorso del processo in tempo ragionevole";

(d) regolare le spese: decidere se gli atti processuali richiesti o proposti e se la produzione probatoria desiderata dalle parti sono cost-effective, ovvero se è osservato il giudizio di proporzionalità.

L'esercizio di questi poteri, che consentano uno spettro decisionale ampio, richiede la preparazione delle corti superiori (Appellate courts) anche nel senso ideologico, le quali non possono esercitare il controllo delle decisioni dei giudici di primo grado senza tenere in conto la teleologia del case management, con il rischio di costringere i giudici ad un approccio timido e reticente. Ciò vuol dire che lo spirito del case management implica un cambio ideologico molto più radicale di quanto si possa comprendere in questo momento di evoluzione della procedura civile.

Nella procedura inglese le parti devono chiedere l'ammissibilità dell'impugnazione di una decisione di case management; tuttavia non è facile ottenere il permesso di impugnare la misura di case management.

\footnotetext{
${ }^{30}$ IVI p. 12.

${ }^{31}$ IBIDEM.
} 
Revista Eletrônica de Direito Processual - REDP.

Rio de Janeiro. Ano 12. Volume 19. Número 2. Maio a Agosto de 2018

Periódico Quadrimestral da Pós-Graduação Stricto Sensu em Direito Processual da UERJ

Patrono: José Carlos Barbosa Moreira (in mem.). ISSN 1982-7636. pp. 84-117

www.redp.uerj.br

Con riferimento all'opportunità di permettere l'impugnazione, bisogna

considerare il contenuto della Practice Direction 52, 4.4, 4.5, a cui fa riferimento Andrews $^{32}$, con la valutazione di seguito riportata:

Case management decisions include decisions made under rule 3.1 (2) [containing a long list of procedural powers] and decision about disclosure, filing of witness statements, or experts reports, directions about the timetable of the claim, adding a party to a claim, and security for costs'. In this context, a decision concerning permission to appeal requires consideration whether 'the issue is of sufficient significance to justify the costs of an appeal', 'the procedural consequences of an appeal (e.g. loss of trial date) outweigh the significance of the case management decision', and whether 'it would be more convenient to determine the issue at or after trial'.

Inequivocabilmente, quindi, le attività di case management richiedono un sistema di disciplina e di sanzione che sia effettivamente applicabile e che non si nutra di clemenza riguardo le parti, in nome della salvezza del processo. È necessario, in realtà, un'esposizione specifica riguardo a tali mezzi (CPR 3.8 (2); 3.1 (2) (f); 3.4(2) (c).

\section{L’importanza della fase preparatoria nel processo civile}

Nel processo civile di common Law, sia inglese che nord americano, sono due le fasi che svolgono ruoli piuttosto rilevanti. Queste fasi sono la pre-action e la pre-trial. La comprensione di questi istituti richiede la necessità di avere ben chiaro che il trial, nel Commom Law, è un momento continuo nel quale si svolge l'attività di indagine, rappresentazione della prova e giudizio della causa. D'altronde, nei sistemi continentali le fasi che precedono l'inizio formale del processo (citazione o ricorso) non hanno quasi alcuna rilevanza.

Nel sistema inglese, i pre-action protocols, menzionate precedentemente, rappresentano un'importante fase di preparazione della causa e dei suoi obiettivi. Da quanto precisato nell'introduzione delle Practice Directions, si può facilmente comprendere che: "Pre-action protocols explain the conduct and set out the steps the court would normally expect parties to take before commencing proceedings for particular types of civil claims".

Gli obiettivi delle "pre-action protocols" (England and Wales) aiutano a capire lo spirito che dà vita al case management; le Practice Directions 3 indicano:

\footnotetext{
${ }^{32}$ ANDREWS, op. cit., p. 13.
} 
Revista Eletrônica de Direito Processual - REDP.

Rio de Janeiro. Ano 12. Volume 19. Número 2. Maio a Agosto de 2018

Periódico Quadrimestral da Pós-Graduação Stricto Sensu em Direito Processual da UERJ

Patrono: José Carlos Barbosa Moreira (in mem.). ISSN 1982-7636. pp. 84-117

www.redp.uerj.br

3. Before commencing proceedings, the court will expect the parties to have exchanged sufficient information to-

(a) understand each other's position;

(b) make decisions about how to proceed;

(c) try to settle the issues without proceedings;

(d) consider a form of Alternative Dispute Resolution (ADR) to assist with

settlement;

(e) support the efficient management of those proceedings; and

(f) reduce the costs of resolving the dispute.

Il processo inglese prevede anche la fase pre-trial, nella quale la preparazione della causa ha già raggiunto un grado di strutturazione più complessa. Questo modo di suddividere il processo è ben distinto dal modo di frammentazione del processo civile continentale. Purtroppo non si può in questa sede procedere con l'analisi delle peculiarità di ogni fase; è importante, però, chiarire che il case management esige una quantità di cognizione all'inizio della domanda, che implica una maggiore importanza della fase introduttiva o preparatoria delle domande.

Riguardo ai "pre-trial hearings", vi sono due importanti udienze: 'case management conference' ('CMCs') e 'pre-hearing review'. Nel pre-trial hearing la produzione di prova orale è eccezionale; nel procedimento ordinario questa udienza è destinata a discutere il contenuto delle dichiarazioni dei testimoni (che sono ridotte a dichiarazioni scritte). È dunque un'udienza di grande utilità, dal momento che possono essere richieste ordinanze provvisorie (interim relief), oppure l'estinzione della domanda dell'attore o di domande/eccezioni del convenuto.

La procedura inglese prevede due forme di disclosure (o due momenti di disclosure): pre-action e pre-trial. L'obiettivo di entrambe è l'accesso alle informazioni importanti; uguaglianza nell'accesso all'informazione; facilitazione o agevolazione del processo di accordo; evitare il processo-trappola (trial by ambush) ${ }^{33}$.

Il procedimento di disclosure è, di regola, assegnato alle seguenti attività: $a$ ) prima di cominciare il processo, ogni parte deve preparare e dare accesso alla lista di documenti sui quali baserà la sua domanda o che possono avere un rapporto con l'interesse dell'altra parte o che possono influenzare la domanda dell'altra parte; si intende, per documento, qualsiasi elemento che possa contenere informazioni ('anything in which information of any description is recorded'); b) l'elemento documentale oggetto di

\footnotetext{
${ }^{33} \mathrm{ID}$, op. cit., p. 19.
} 
Revista Eletrônica de Direito Processual - REDP.

Rio de Janeiro. Ano 12. Volume 19. Número 2. Maio a Agosto de 2018

Periódico Quadrimestral da Pós-Graduação Stricto Sensu em Direito Processual da UERJ

Patrono: José Carlos Barbosa Moreira (in mem.). ISSN 1982-7636. pp. 84-117

www.redp.uerj.br

disclosure non può essere diffuso tra altri soggetti ${ }^{34}$. Le Practice Directions regolano in tal senso l'uso successivo delle informazioni ottenute nella Disclosure (PD, 31.22):

(1) A party to whom a document has been disclosed may use the document only for the purpose of the proceedings in which it is disclosed, except where -

(a) the document has been read to or by the court, or referred to, at a hearing which has been held in public;

(b) the court gives permission; or

(c) the party who disclosed the document and the person to whom the document belongs agree.

(2) The court may make an order restricting or prohibiting the use of a document which has been disclosed, even where the document has been read to or by the court, or referred to, at a hearing which has been held in public.

È nella fase introduttiva o preparatoria, infatti, che si prendono le decisioni più importanti riguardo al percorso processuale e che possono determinare la riduzione della durata del processo.

Di conseguenza, la fase preparatoria del processo civile ha acquisito una singolare importanza. Più che la mera presentazione di una petizione, di una citazione o di un ricorso, si tratta di preparare la domanda in senso più ampio.

È proprio in questo momento del processo che ci sono stati significativi cambiamenti nel corso delle ultime riforme processuali degli Stati europei.

Infatti, la trattazione iniziale del processo, se svolta in modo tale che il giudice possa fissare i fatti e le questioni controverse, può eliminare tutto ciò che non è in realtà vero oggetto del processo. La fase preparatoria moderna ha come obiettivo quello di identificare i punti di discussione e i bisogni probatori.

In quest'ottica, dunque, la linea evolutiva del processo civile europeo si presenta come l'idea di dare una sempre più significativa importanza alla fase preparatoria, nella quale il giudice esercita importanti poteri di adeguamento del rito all'oggetto della lite: "presenza attiva del giudice al fine di determinare il quod decidendum e il quod probandum"35.

Questi sono alcuni aspetti più rilevanti del c.d. active case management in Inghilterra e in Galles.

\footnotetext{
${ }^{34}$ È il caso di applicazione della 'Implied Undertaking Rule'. Una regola inglese originata nel mezzo del secolo XIX, che riguarda l'attività di protezione rispetto ai dati oggetto di disclosure. La norma stabilisce il dovere di non usare i documenti ad altri fine che non sia quello di essere usati nel ambito della specifica domanda giudiziale. La corte può anche decidere di permettere l'uso dei documenti in altre ambiti.

${ }^{35}$ FICCARELLI, op. cit., p. 13.
} 
Revista Eletrônica de Direito Processual - REDP.

Rio de Janeiro. Ano 12. Volume 19. Número 2. Maio a Agosto de 2018

Periódico Quadrimestral da Pós-Graduação Stricto Sensu em Direito Processual da UERJ

Patrono: José Carlos Barbosa Moreira (in mem.). ISSN 1982-7636. pp. 84-117

www.redp.uerj.br

\section{La questione dei costi del processo}

Il 1 aprile 2013 è entrata in vigore in Inghilterra e in Galles la Jackson Reforms. Tale riforma ha revisionato il sistema di costi giudiziarie e ha modificato il case management.

Innanzitutto, è necessario chiarire che in Inghilterra non esiste l'obbligo di assistenza legale, tuttavia non si può prescindere dall'assistenza legale dinanzi ad un sistema estremamente tecnico senza assumere il rischio di essere assolutamente pregiudicato.

In secondo luogo, le spese giudiziali in Inghilterra sono mediamente otto volte superiori a quelle italiane ${ }^{36}$, ma possono raggiungere valori significativi senza un rapporto diretto con il valore della causa, bensì con la quantità e la qualità dell'attività difensiva. Riguardo a tale aspetto, Zuckerman afferma: "The cost of litigation in England is unpredictable, excessive and disproportionate. It seems to make little difference, in this regard, whether the dispute is complicated or simple, important or trivial. Even a dispute which is speedily resolved can run up huge litigation costs" ${ }^{\prime 37}$.

Il problema dei costi giudiziari era già stato una delle principali ragioni della Riforma Woolf, il quale ebbe a ritenere che la causa dei altissimi costi non fosse tanto la complessità della procedura quanto piuttosto la 'uncontrolled nature of the litigation process $^{38}$. Secondo lui, la soluzione, già tante volte qui menzionata, era il controllo del giudice.

All'esito dell'ampio dibattito che ha preceduto la Riforma Woolf, sono state stabilite, come già menzionato in antecedenza, due forme di controllo. La prima, quella del controllo legale, ovvero una procedura stretta che garantisce l'avanzamento del processo. La seconda, quella del controllo del giudice, che d'ora in poi è il manager del processo.

Modificando la tradizionale divisione di attribuzioni del sistema adversariale, Lord Woolf ha così concentrato nelle attività dei giudici il controllo della lite. L'aspetto che attira molta attenzione è il fatto che l'obiettivo di questa scelta lungimirante era essenzialmente

\footnotetext{
${ }^{36}$ MARINARI M., La riforma Jackson e la disciplina delle spese nel processo civile inglese. Disponibile su: http://www.questionegiustizia.it/articolo/la-riforma-jackson-e-la-disciplina-delle-spese-nel-processo-civileinglese 31-05-2014.php, accesso il 17 ottobre 2017.

${ }^{37}$ ZUCKERMANN, Lord Woolf's Access to Justice: Plus Ça Change, Modern Law Review, cit., p. 1.

${ }^{38}$ IVI, p. 2.
} 
Revista Eletrônica de Direito Processual - REDP.

Rio de Janeiro. Ano 12. Volume 19. Número 2. Maio a Agosto de 2018

Periódico Quadrimestral da Pós-Graduação Stricto Sensu em Direito Processual da UERJ

Patrono: José Carlos Barbosa Moreira (in mem.). ISSN 1982-7636. pp. 84-117

www.redp.uerj.br

quello di controllare la quantità di lavoro che gli avvocati avrebbero potuto o dovuto svolgere in ogni tipo di domanda, e dunque controllare i costi giudiziali ${ }^{39}$.

La chiave di analisi gira intorno all'eccessiva libertà concessa agli avvocati nel protrarre o allungare il processo. Pertanto, combattere l'abuso è stata (e continua ad essere) la motivazione principale delle riforme inglesi.

Al fine di comprendere la dinamica dei cambiamenti apportati dalla Riforma Woolf e dalla Jackson Reforms, e quindi di affrontare con i dovuti strumenti lessicali il tema, occorre anzitutto fornire la nuova terminologia che tali riforme adottano. E così, come riportato dall'analisi del Marinari ${ }^{40}$ :

Fixed Costs: per domande di minor valore (recupero di somme o di beni, incidenti stradali, materia di esecuzione); Onorari- Costs capping (CCOs): "an order limiting the amount of future costs (including disbursements) which a party may recover pursuant to an order for costs subsequently made"; Small Claims: nessuna spesa legale (tuttavia è prevista la condanna della parte soccombente al rimborso delle spese materiali sostenute dalla controparte); Costs order in favour or against a non-party: soggetti diversi dalle parti possono essere sanzionati con una condanna alle spese (soggetti che abbiano finanziato la parte soccombente o compiuto altre attività); Waisted Costs: la condotta ingiustificabile dell' avvocato (improper, unreasonable, negligent) può essere sanzionata se ha danneggiato la parte soccombente, anche nel caso di mancata informazione al cliente, specialmente nel caso di hopeless claims. Criteri di liquidazione delle spese: Standard Costs - coprono una percentuale però non la totalità delle spese legali; Indemnity Costs senso sanzionatorio e che può giungere a coprire $100 \%$ della richiesta contenuta nel Bill of Costs. Conditional Fee Agreements (CFA's): la parte può essere esonerata dal pagamento dell'onorario al proprio avvocato; (non si tratterebbe di un equivalente delle Contingency Fees americane). Normalmente si prevede una percentuale calcolata sull'importo liquidato al favore della parte (success fee): "come nel meccanismo delle Contingency Fees, ma che si aggiunge, in questo caso, all'importo delle spese liquidate a favore della parte vincitrice (receiving party), e non invece, come avviene per le contingency fees, detraendo il compenso dell'avvocato dall'ammontare del risarcimento del danno o comunque della

\footnotetext{
${ }^{39}$ IVI, p. 3.

${ }^{40}$ MARINARI M., La riforma Jackson e la disciplina delle spese nel processo civile inglese. Disponibile su: http://www.questionegiustizia.it/articolo/la-riforma-jackson-e-la-disciplina-delle-spese-nel-processo-civileinglese 31-05-2014.php, accesso il 17 ottobre 2017.
} 
Revista Eletrônica de Direito Processual - REDP.

Rio de Janeiro. Ano 12. Volume 19. Número 2. Maio a Agosto de 2018

Periódico Quadrimestral da Pós-Graduação Stricto Sensu em Direito Processual da UERJ

Patrono: José Carlos Barbosa Moreira (in mem.). ISSN 1982-7636. pp. 84-117

www.redp.uerj.br

somma attribuita alla parte vincitrice"41. ATE (After The Event) Insurance Premium:

premio della speciale assicurazione stipulata dalla parte vincitrice per coprirsi dal rischio di soccombenza, che sarà rimborsato dalla parte soccombente. Additional liability: l'insieme di tali costi aggiuntivi costituisce l'attribuibile alla parte soccombente ${ }^{42}$.

Di tutto ciò che è stato detto risulta evidente che la questione delle spese è diventata il tema centrale delle riforme della procedura inglese ${ }^{43}$. Secondo quanto spiega Comoglio, le Rules of the Supreme Court anteriori alle attuali $C P R$, permetteva alla parte vincitrice di ottenere dalla parte soccombente il rimborso dei costi e in genere delle spese «necessary or proper for the attainment of justice ». Ciò rappresenterebbe il criterio valido dal 1883 al 1986, quando fu introdotto il suddetto criterio indennitario («assessment on the indemnity basis»), affiancato al criterio di «assessment on the standard basis», il quale determinava la ripetibilità di costi ragionevoli, criterio adottato nella prima versione della CPR. Lì sarebbe presente già l'idea di proporzionalità dei costi. Secondo Comoglio, “il recente Update delle $\mathrm{CPR}$ inglesi riconosce il principio di proporzionalità dei costi nel processo civile ("principle of proportionality in relation to civil procedure ») quale nozione autonoma e fondante ${ }^{, 44}$.

La proporzionalità dei costi acquista indipendenza ed autonomia nell'ambito del overridding objective. La proporzionalità acquista prevalenza sul principio di necessità, concedendo al giudice inglese una dimensione di discrezionalità che prima non possedeva, specialmente perché tale controllo è richiesto all'inizio della domanda, in modo preventivo.

Il giudice del case management inglese ha un ruolo attivo/determinante anche riguardo ai costi del processo. Questo controllo non è solo a posteriori ma anche preventivo.

Nella Part 3 del CPR sono elencati gli aspetti principali del nuovo controllo sui costi del processo, dove si determina:

\footnotetext{
${ }^{41}$ MARINARI M., La riforma Jackson e la disciplina delle spese nel processo civile inglese. Disponibile su: http://www.questionegiustizia.it/articolo/la-riforma-jackson-e-la-disciplina-delle-spese-nel-processo-civileinglese 31-05-2014.php, accesso il 17 ottobre 2017.

${ }^{42}$ IBIDEM.

${ }^{43}$ COMOGLIO L. P., Giustizia (non) a tutti i costi. significativo «update» delle civil procedure rules inglesi e suggestioni sistematiche per la riforma del processo civile, in Riv. Trim. di dir. civ. e proc. civ., fasc.1, 2014, pag. 145.

44 IBIDEM.
} 
Revista Eletrônica de Direito Processual - REDP.

Rio de Janeiro. Ano 12. Volume 19. Número 2. Maio a Agosto de 2018 Periódico Quadrimestral da Pós-Graduação Stricto Sensu em Direito Processual da UERJ Patrono: José Carlos Barbosa Moreira (in mem.). ISSN 1982-7636. pp. 84-117 www.redp.uerj.br

(a) ciascuna parte prima della "first case management conference" offre un budget contenente l'indicazione dei possibili costi per tutta la causa, compreso il trial. Questo preventivo sarà discusso tra le parti, nel corso di un'udienza di Case Management.

(b) il giudice può emettere "costs management order" al fine di controllare la correttezza dei budget, che resterà vincolante per il procedimento, regolando i costi, la durata e la complessità della fase pre-trial.

(c) il giudice può fissare apposite conference alla gestione dei costi;

(d) nella liquidazione delle spese, il giudice utilizza come principale riferimento il budget approvato o concordato, "non potendosene discostare se non in presenza di una "good reason".

Nella section III della part 3 del CPR troviamo il "Costs capping order", "an order limiting the amount of future costs (including disbursements) which a party may recover pursuant to an order for costs subsequently made" (Rule 3.19).

Nel sistema inglese la liquidazione delle spese viene frequentemente effettuata da un altro giudice, attualmente chiamato Costs Judge o Costs Officer, dopo l'introduzione delle CPR); in precedenza si chiamava Taxing Master (prima delle CPR) ${ }^{45}$.

Part 44 delle CPR: (numerose eccezioni alla regola generale del principio di soccombenza) - specifici elementi per la valutazione del comportamento delle parti: a) ruolo attribuito alla condotta preprocessuale; b) al rispetto dei pre-action protocol; c) alla ragionevolezza nell'agire o nel resistere in giudizio; d) "al modo con cui è stata espletata la difesa; e) e all'eventuale ingiustificato aggravamento dell'attività processuale ad opera della parte vincitrice.

Il sistema di gestione delle spese in Inghilterra e in Galles non osserva più la semplice regola della soccombenza, ovvero chi perde paga, tantomeno la regola della causalità, ma ha un sistema proprio che si nutre dell' idea di responsabilità riguardo ai costi.

In tal senso, Comoglio afferma che la disciplina inglese può essere estesa al regime italiano delle spese processuali, in primo luogo perché la riforma della legge n. 69 del 2009 ha approssimato i regimi ${ }^{46}$, in secondo luogo perché è possibile visualizzare nel

\footnotetext{
${ }^{45}$ IBIDEM.

46 Difatti, l'art. 92, comma 1, consente al giudice di escludere la ripetizione di parte delle spese sostenute dalla parte vincitrice qualora le ritenga «eccessive o superflue ».
} 
Revista Eletrônica de Direito Processual - REDP.

Rio de Janeiro. Ano 12. Volume 19. Número 2. Maio a Agosto de 2018

Periódico Quadrimestral da Pós-Graduação Stricto Sensu em Direito Processual da UERJ

Patrono: José Carlos Barbosa Moreira (in mem.). ISSN 1982-7636. pp. 84-117

www.redp.uerj.br

contesto italiano la responsabilità per spese collegata alla violazione dei generali principi di colpevolezza (art. 2043, C.C), e alla violazione dei principi di lealtà e probità, tesi già sostenuta $^{47}$.

L'autore sostiene, tra l'altro, che il giudice potrebbe anticipare il controllo delle attività processuali eccessive o superflue, facendo attenzione all' economia processuale $\mathrm{e}$ alla ragionevole durata; in tal senso i poteri direttivi del giudice si conformano a un'idea manageriale del processo ${ }^{48}$. In tale direzione, a titolo di esempio Comoglio difende la ripetibilità delle spese nel caso delle memorie di cui al comma 6 dell'art. 183 c.p.c, quando siano depositate o siano mera ripetizione, prive di utilità-

La part 44 introduce significative eccezioni alla regola del principio di soccombenza, eccezioni fondate sul comportamento della parte. Il comportamento preprocessuale raggiunge importanza e viene valutato secondo la ragionevolezza delle scelte delle parti e quanto queste scelte hanno influenzato il percorso/l'attività processuale.

Come visto precedentemente, la gestione delle spese possiedono un ruolo rilevante nell'active case management inglese; tuttavia, evitare lo spreco di risorse è una preoccupazione universale della procedura civile, indipendentemente dal ruolo che le spese giudiziarie svolgono; cioè a dire, indipendentemente se raggiungono o no somme sproporzionate.

\section{Case management, Pre-trial e Discovery negli Stati Uniti}

Il case management è un istituto di common Law presente anche nel sistema nord americano. Secondo quanto riferito da Langbein ${ }^{49}$, riguardo agli Stati Uniti, il "Managerial judging" nasce inizialmente nelle Federal Courts (anni 70), come risposta del sistema al

\footnotetext{
${ }^{47}$ V. COMOGLIO L. P., "Giustizia non a tutti i costi", cit., nota 54.

${ }^{48}$ IVI, pag. 145: "Certo, un problema di fondo al riguardo consisterebbe nella definizione dei limiti di realizzazione di tale controllo. Come si è accennato prima, nell'ordinamento inglese il giudice, in nome del principio di proporzionalità, può sacrificare non solo le attività superflue, ma anche quelle necessarie ove sproporzionate al valore della controversia. Si tratterebbe di una valutazione discrezionale per contro non coerente, in ragione della sua ampiezza, con il quadro normativo italiano. Ciò in particolare con riferimento all'ammissione di mezzi istruttori; ammissione collegata dal nostro codice a meri criteri di rilevanza $\mathrm{o}$, in alcuni e tassativi casi (artt. 118 e 210 c.p.c.), di necessità (57). È pertanto da intendersi che, pur ipotizzando forme di controllo preventivo sulle spese, il giudice italiano non potrà escludere un mezzo di prova, se necessario ai fini della decisione, sulla sola base dell'eventuale sproporzione con il valore della controversia". ${ }^{49}$ LANGBEIN J. H., The German Advantage in Civil Procedure. The University of Chicago Law Review. Volume 52, number 4, Fall 1985, p. 825.
} 
Revista Eletrônica de Direito Processual - REDP.

Rio de Janeiro. Ano 12. Volume 19. Número 2. Maio a Agosto de 2018

Periódico Quadrimestral da Pós-Graduação Stricto Sensu em Direito Processual da UERJ

Patrono: José Carlos Barbosa Moreira (in mem.). ISSN 1982-7636. pp. 84-117

www.redp.uerj.br

costante aumento delle liti complesse.

A tale scopo, negli Stati Uniti sorge il Manual for Complex Litigation. Si badi, però, che le attribuzioni delle managerial judging non vengono affiancate ai casi complessi:

The Manual for Complex Litigation was created to deal with these cases, but because complexity is a matter of degree, managerial judging was hard to confine to the Big Case. The Manual identifies antitrust, securities, mass disaster, product liability,class action, and multiparty cases, among others, as typical. 122 In cases with many parties and many issues, the feeling grew that court-centered control was needed to prevent the confusion and duplication that would result if the adversaries were "left to themselves, each pursuing the course that is most favorable to his particular client. 123 Accordingly, "[t]he essence" of what the Manual propounds "is the exercise of judicial control over complex litigation plus a positive plan for discovery and pretrial preparation. '12 4.

Il Manual for Complex Litigation stabilisce misure che conferiscono al giudice maggiori poteri di interferenza e controllo sulla produzione delle prove, al contrario del potere ordinario che viene relegato alle parti, nel sistema adversariale:

(1) The judge uses pretrial conferences to explore the case with counsel and to identify key issues.12 5 (2) The judge is expected to promote settlement from the earliest opportunity. 26 (3) The judge also helps sharpen the issues. "To the extent feasible the judge should narrow the issues in the course of the first pretrial conference and limit discovery accordingly. 127 (4) Issue definition leads to the regulation of discovery." I poteri di controllo della discovery sono più tardi codificati nel Federal Rules of Civil Procedure (rule 26 (f).

La "case management conference" è normalmente stabilita dopo che una domanda è iniziata (a complaint and answer are filed). Nell'udienza, il giudice emana una Case Management Order, ove sono determinati i procedimenti di discovery (processo, ampiezza e limitazioni), pre-trial conferences e trial.

La Discovery è essenzialmente una procedura pre-trial, un processo di investigazione formale, che permette alle parti di ottenere prove dall'altra parte per mezzo degli strumenti procedurali; tali strumenti sono interrogatori, domanda di produzione di documenti, investigazioni, esame fisico, domanda per ammissioni di fatti (request for admissions and depositions). Negli Stati Uniti, secondo quanto stabilito dal n. 45 delle Federal Rules of civil procedure, anche le non-parties possono essere chiamate a testimoniare sub poenas. 
Revista Eletrônica de Direito Processual - REDP.

Rio de Janeiro. Ano 12. Volume 19. Número 2. Maio a Agosto de 2018

Periódico Quadrimestral da Pós-Graduação Stricto Sensu em Direito Processual da UERJ

Patrono: José Carlos Barbosa Moreira (in mem.). ISSN 1982-7636. pp. 84-117

www.redp.uerj.br

Qualsiasi elemento che possa servire come mezzo di prova nel processo può

essere oggetto di discovery. Ciò significa che, anche elementi sui quali vi è dubbio riguardo alla propria rilevanza giuridica, possono essere oggetto di una richiesta di discovery. Tuttavia, possono esservi delle informazioni protette, come ad esempio il prodotto del lavoro e le informazioni privilegiate (privileged information).

Nella legislazione nord americana, discovery è un procedimento avviato dalle parti, con poca "judicial oversight". Secondo le "Federal Rules of Civil Procedure", l'attore (plaintiff), inizia la conferenza (conference) tra le parti, dopo che la citazione/ricorso (complaint: the first pleading of the plaintiff in a civil action, stating the cause of action) viene notificata, presentata (served) al convenuto, pianificando il processo di discovery.

Il procedimento di discovery viene negato nel caso in cui l'informazione ricercata non è necessaria a una domanda giudiziale. Dopo che il procedimento di discovery viene finalizzato, se non è ancora stata fissata la pre-trial conference, l'avvocato deve chiedere al Tribunale di fissare una data per la pre-trial conference e il trial.

La fase del pre-trial è caratterizzata per una non delimitazione assoluta del suo oggetto. Ciò è conseguenza delle regole che stabiliscono che la forma del "complaint" sia semplice e non molto prolissa. Federal Rules of Civil Procedure $8^{50}$ (a) and $8(\mathrm{e})$ stabilisce:

${ }^{\mathbf{5 0}}$ Rule 8. General Rules of Pleading

(a) CLAIM FOR RELIEF. A pleading that states a claim for relief must contain:

(1) a short and plain statement of the grounds for the court's jurisdiction, unless the court already has jurisdiction and the claim needs no new jurisdictional support;

(2) a short and plain statement of the claim showing that the pleader is entitled to relief; and

(3) a demand for the relief sought, which may include relief in the alternative or different types of relief.

(b) DEFENSES; ADMISSIONS AND DENIALS.

(1) In General. In responding to a pleading, a party must:

(A) state in short and plain terms its defenses to each claim asserted against it; and

(B) admit or deny the allegations asserted against it by an opposing party.

(2) Denials-Responding to the Substance. A denial must fairly respond to the substance of the allegation.

(3) General and Specific Denials. A party that intends in good faith to deny all the allegations of a pleading including the jurisdictional grounds - may do so by a general denial. A party that does not intend to deny all the allegations must either specifically deny designated allegations or generally deny all except those specifically admitted.

(4) Denying Part of an Allegation. A party that intends in good faith to deny only part of an allegation must admit the part that is true and deny the rest.

(5) Lacking Knowledge or Information. A party that lacks knowledge or information sufficient to form a belief about the truth of an allegation must so state, and the statement has the effect of a denial.

(6) Effect of Failing to Deny. An allegation - other than one relating to the amount of damages - is admitted if a responsive pleading is required and the allegation is not denied. If a responsive pleading is not required, an allegation is considered denied or avoided.

(c) AFFIRMATIVE DEFENSES. 
Revista Eletrônica de Direito Processual - REDP.

Rio de Janeiro. Ano 12. Volume 19. Número 2. Maio a Agosto de 2018

Periódico Quadrimestral da Pós-Graduação Stricto Sensu em Direito Processual da UERJ

Patrono: José Carlos Barbosa Moreira (in mem.). ISSN 1982-7636. pp. 84-117

www.redp.uerj.br

A complaint shall contain (a) CLAIM FOR RELIEF. A pleading that states a claim for relief must contain: (1) a short and plain statement of the grounds for the court's jurisdiction, unless the court already has jurisdiction and the claim needs no new jurisdictional support; (2) a short and plain statement of the claim showing that the pleader is entitled to relief; and (3) a demand for the relief sought, which may include relief in the alternative or different types of relief.

Semplice, conciso, diretto, senza aspetti tecnici. Pertanto, questa apertura della fase iniziale permette alle parti di conformare la propria domanda nella durata della discovery.

\title{
5. Case management, il ruolo del giudice e le risorse dell'apparato giudiziario
}

Ma che cosa è, essenzialmente, l'active case management? Nella sintesi di Paolo Biavati, è il "dedicare al singolo giudizio tempi e risorse adeguati, rispetto sia alle esigenze di quel caso, ma anche a quelle complessive del carico giudiziario" ${ }^{„ 51}$.

Il giudice è in tal senso un efficace organizzatore del processo $^{52} \mathrm{e}$ il suo ruolo attivo si realizzerebbe precisamente nei seguenti fronti:

\begin{abstract}
nell'incoraggiare le parti alla reciproca cooperazione nella conduzione del processo; nell'identificare le questioni controverse;

nell'individuare prontamente le questioni che richiedono un'istruzione approfondita e quali, invece, possono essere oggetto di decisione sommaria; nel decidere l'ordine in cui le questioni debbono essere risolte;

nell'incoraggiare le parti all'uso di strumenti alternativi di risoluzione delle controversie qualora lo consideri opportuno e nel facilitarne l'espletamento;
\end{abstract}

(1) In General. In responding to a pleading, a party must affirmatively state any avoidance or affirmative defense, including: $\bullet$ accord and satisfaction; $\bullet$ arbitration and award; $\bullet$ assumption of risk; $\bullet$ contributory negligence; $\bullet$ duress; $\bullet$ estoppel; $\bullet$ failure of consideration; $\bullet$ fraud; • illegality; • injury by fellow servant; • laches; $\bullet$ license; $\bullet$ payment; • release; $\bullet$ res judicata; $\bullet$ statute of frauds; $\bullet$ statute of limitations; and $\bullet$ waiver.

(2) Mistaken Designation. If a party mistakenly designates a defense as a counterclaim, or a counterclaim as a defense, the court must, if justice requires, treat the pleading as though it were correctly designated, and may impose terms for doing so.

(d) Pleading to Be Concise and Direct; Alternative Statements; InCONSistency.

(1) In General. Each allegation must be simple, concise, and direct. No technical form is required.

(2) Alternative Statements of a Claim or Defense. A party may set out 2 or more statements of a claim or defense alternatively or hypothetically, either in a single count or defense or in separate ones. If a party makes alternative statements, the pleading is sufficient if any one of them is sufficient.

(3) Inconsistent Claims or Defenses. A party may state as many separate claims or defenses as it has, regardless of consistency.

(e) Construing Pleadings. Pleadings must be construed so as to do justice.

${ }^{51}$ BIAVATI P., Osservazioni sulla Ragionevole Durata del Processo di Cognizione. Riv. trim. dir. proc. civ., fasc. 2, 2012, pag. 475.

${ }_{52}$ BIAVATI P., Appunti sulla Struttura della Decisione e L'ordine delle questioni, Riv. trim. dir. proc. civ., fasc. 4, 2009, pag. 1301 . 
Revista Eletrônica de Direito Processual - REDP.

Rio de Janeiro. Ano 12. Volume 19. Número 2. Maio a Agosto de 2018

Periódico Quadrimestral da Pós-Graduação Stricto Sensu em Direito Processual da UERJ

Patrono: José Carlos Barbosa Moreira (in mem.). ISSN 1982-7636. pp. 84-117

www.redp.uerj.br

nell'aiutare le parti a transigere in tutto o in parte la causa;

nel considerare se i probabili effetti positivi derivanti dall'adozione di un particolare provvedimento giustifichino i costi che da esso derivano;

nel dar direttive affinché le udienze si svolgano rapidamente ma efficientemente;

nel fissare un calendario processuale o comunque controllando la progressione della causa ${ }^{53}$.

Nel processo inglese, il giudice all'interno del processo possedeva un ruolo sostanzialmente passivo ${ }^{54}$, nonostante esercitasse poteri di direzione del processo, un ruolo in consonanza con il modello del liberalismo classico e dell'individualismo economico ${ }^{55}$.

Quali sono, invece, i poteri del giudice che vanno oltre la normale direzione del processo e che conferiscono un ulteriore grado nel ruolo di direzione del processo, già messo in esecuzione nei sistemi di civil law?

Il giudice esercita un ruolo attivo mediante i poteri di imposizione della comparizione personale delle parti, nel potere di stabilire i termini nell'ambito del processo, oppure attraverso accordi conciliativi o aspetti processuali della concretizzazione delle tutele e ordinanze provvisorie.

L'idea di active ${ }^{56}$ case management ha come traguardo quello di ottenere un'adeguata trattazione, la quale dipende da due elementi: rito efficiente a produrre una decisione giusta ${ }^{57}$ e che riproduca un corretto rapporto di bilancio tra costi e benefici. Trattasi, in realtà, di un tema che richiede un esame approfondito e intorno al quale si concentrano diverse discussioni, come ad esempio il rapporto tra processo giusto e i ricorsi disponibili nell'apparato giudiziario. Per meglio dire, quale è il giusto rito che il sistema può offrire a ogni domanda? Riteniamo che tale aspetto richiama un'analisi del rapporto tra il giusto processo rispetto alla durata ragionevole.

\footnotetext{
${ }^{53}$ FICCARELLI, op. cit., p. 34.

${ }^{54}$ DONZELLI, op. cit. , p. 5.

${ }^{55}$ IBIDEM.

${ }^{56}$ BONCI, op. cit., p. 228: "Although Italian civil procedure acknowledges the notion of case management, the Italian system is in fact still a long way from embracing the notion of "active case management". The concept of "active" involvement refers precisely to the ways in which judges make use of the managerial powers conferred to them. Tellingly, Senior Master Robert Turner (a former High Court judge in London) has identified "actively" as "the [single] word that changed the [English] civil courts".

${ }^{57}$ Vedi BIAVATI P., Appunti Sulla Struttura Della Decisione E L'ordine Delle Questioni, cit., pag. 1301: "La sostanza della lite è ciò su cui si esercita la domanda sociale di giustizia, ciò su cui si confrontano il torto e la ragione, ciò per cui è necessario l'intervento sostitutivo del giudice. A nessuno sfugge l'importanza del rito ed il metodo del contraddittorio ha rilevanza costituzionale (5): tuttavia, è doveroso ricordarsi che il rito ha una funzione soltanto strumentale ed è una condizione di garanzia per il corretto esercizio dialettico della funzione decisoria, che però tende alla risoluzione del conflitto sul merito".
} 
Revista Eletrônica de Direito Processual - REDP.

Rio de Janeiro. Ano 12. Volume 19. Número 2. Maio a Agosto de 2018

Periódico Quadrimestral da Pós-Graduação Stricto Sensu em Direito Processual da UERJ

Patrono: José Carlos Barbosa Moreira (in mem.). ISSN 1982-7636. pp. 84-117

www.redp.uerj.br

Una parte della dottrina processuale sostiene che non si raggiunge un'adeguata

trattazione con uno schema predeterminato di rito processuale, ma è necessaria una struttura elastica che permetta la differenziazione dei riti $^{58}$. La classica divisione tra $\mathrm{i}$ prodotti: Taylor made e prêt-à-porter.

L'active case management concede al giudice discrezionalità anche in quelle situazioni dove di solito non c'è mai stata o ce n'è stata pochissima. Da ciò deriva l'influsso del principio di proporzionalità ${ }^{59}$, del quale l'adattabilità, la flessibilità e l'elasticità non sono altro che manifestazioni.

Oltre a quanto finora esposto, bisogna altresì considerare che le risorse dell'assetto giudiziario non sono illimitate. Infatti, gli sforzi e le energie disponibili devono essere distribuite con ragionevolezza e bisogna misurare quanto si può dare ad ogni processo in modo isonomico, considerando il tempo e una durata ragionevole. In tal modo, è necessario ribadire che "vi deve essere proporzionalità fra le risorse complessive di sistema e quelle dedicate ad un singolo processo" $" 60$.

La ragionevole durata dipende dalla quantità di risorse disponibili riguardo ad ogni processo, dal periodo di tempo necessario ad una trattazione adeguata, dalla definizione di trattazione adeguata, prendendo in considerazione la offerta delle risorse e la sua dinamica, e deve tendere a proteggere il nucleo del diritto di difesa, senza che esso sia troppo esasperato o violato nella sua estensione.

Di conseguenza, cambia profondamente la valutazione della nozione di economia processuale, di procedura adeguata e diventa importante conoscere le caratteristiche di ogni sistema e le sue risorse, più che altro si esige un accurato rapporto tra la teoria e la materialità delle risorse, nel senso di evitare un'astrazione sbagliata, come spesso succede:

Il secondo pericolo segnalato da Pietro de Francisci, seguace in questo del Gény, è la tendenza della dogmatica a una “obiettivazione assoluta e immutabile

\footnotetext{
${ }^{58}$ Secondo FICCARELLI, op. cit., p. 112: “l'esigenza di un'adeguata trattazione delle cause non può essere efficacemente perseguita disegnando uno schema rigido di processo concepito come universalmente valido per tutti i tipi di controversie. Quell'obiettivo esige strutture elastiche al cui interno vi sia spazio per la differenziazione dei modelli di trattazione delle cause in funzione dei loro caratteri peculiari. L'elasticità dunque come strategia (o scelta) fondamentale per avere un processo che funzioni".

59 ANDREWS, op. cit., p. 10: “Tracks: The allocation of cases to different types of first instance court (county court or High Court) and to different systems of procedure (known as 'tracks') under the CPR (1998) is founded on the principle of jurisdictional proportionality: that litigation must be tailored to the size and nature of the dispute".

${ }^{60}$ Come ha detto BIAVATI P., Osservazioni Sulla Ragionevole Durata del Processo di Cognizione. cit., p. 474, "Si potrebbe forse dire, con un'espressione cara ad un valente autore, che vi deve essere proporzionalità fra le risorse complessive di sistema e quelle dedicate ad un singolo processo".
} 
Revista Eletrônica de Direito Processual - REDP.

Rio de Janeiro. Ano 12. Volume 19. Número 2. Maio a Agosto de 2018 Periódico Quadrimestral da Pós-Graduação Stricto Sensu em Direito Processual da UERJ Patrono: José Carlos Barbosa Moreira (in mem.). ISSN 1982-7636. pp. 84-117 www.redp.uerj.br

di quei concetti, ad una limitazione di categorie, attraverso una serie di processi di logica astratta, nei quali si trascura molte volte lo scopo razionale e pratico degli istituti, per conservarne soltanto l'elemento concettuale staccato dalla natura e dalla vita. ${ }^{61}$

Del resto, bisogna concordare con ciò che afferma Paolo Biavati: "La nozione di economia processuale, invalsa e talvolta fin troppo utilizzata, assume qui una valenza diversa, nel senso che esce da una visione atomistica dei processi e conduce ad una considerazione della globalità del carico di lavoro" $" 62$.

In tal senso, il diritto di difesa deve essere osservato e garantito nella misura in cui conserva integro il suo nucleo. Come è stato già detto: "Insomma, non si devono confondere, nell'esercizio della difesa, la quantità e la qualità "63.

L'adeguata trattazione delle liti ha come tertium comparationis la ragionevole durata; in questo modo tutti i meccanismi di concentrazione ed accelerazione dei processi devono raggiungere l'obiettivo di realizzare il valore della ragionevole durata. Lo spazio necessario alla difesa nel processo è quello che impedisce l'uso abusivo del processo o l'uso non necessario dei meccanismi di attivazione della tutela giurisdizionale.

Le riforme italiane ci portano diversi esempi di tale impostazione. Ad esempio, l'art. 702-ter, comma $5^{\circ}$ e l'art. 669-sexies, comma $1^{\circ}$, c.p.c., hanno come nucleo inderogabile soltanto il rispetto del contraddittorio. Le scelte del legislatore attuale ci conducono alla sommarizzazione, con cognizione piena e oggetto dell'indagine che segue.

\section{La sommarizzazione e la cognizione completa}

L'idea che il processo sommario (procedura semplificata) non possa portare ad una decisione di cognizione piena è stata già rifiutata dalla realtà dei diversi sistemi processuali, incluso quello italiano (si veda il processo sommario di cognizione) ${ }^{64}$. La semplificazione e l'assenza di predeterminazione o deformalizzazione del rito non

\footnotetext{
${ }^{61}$ COSTABILE F., Storicità del Diritto Romano e Creatività Dogmatica per il Diritto Moderno, in Atena e Roma alle origini della democrazia moderna, a cura di ID, Giappichelli Editore, p. 142.

${ }^{62}$ BIAVATI P., Appunti Sulla Struttura Della Decisione E L'ordine Delle Questioni, cit., pag. 1301.

${ }^{63}$ ID., Osservazioni Sulla Ragionevole Durata Del Processo Di Cognizione, cit., pag. 475.

${ }^{64}$ Vide PORRECA P., Il procedimento sommario di cognizione: un rito flessibile, pag. 823, e LUPOI, M. A., Sommario (Ma non Troppo), pag. 1225.
} 
Revista Eletrônica de Direito Processual - REDP.

Rio de Janeiro. Ano 12. Volume 19. Número 2. Maio a Agosto de 2018

Periódico Quadrimestral da Pós-Graduação Stricto Sensu em Direito Processual da UERJ

Patrono: José Carlos Barbosa Moreira (in mem.). ISSN 1982-7636. pp. 84-117

www.redp.uerj.br

impediscono l'accertamento completo della lite ${ }^{65}$. Un procedimento sommario non implica

necessariamente cognizione sommaria. Attualmente, la dottrina più autorevole evidenzia

che i processi sommari possono essere suddivisi in due categorie: processi sommari perché semplificati e processi sommari perché a cognizione sommaria ${ }^{66}$.

Le controversie non sono uguali ed oltre ai concetti di easy case e complex litigation, si possono fare altre classificazioni, prendendo in considerazione un'ampia possibilità di modelli meno rigidi di istruzione e conclusione del processo e che possono riguardare processi di massa, di natura amministrativa, tributaria, ambientale.

Il giusto processo non è altro che la salvaguardia dei principi costituzionali posti in tema di esercizio della funzione giurisdizionale ${ }^{67}$. Il problema che si pone sempre è quanta fiducia ha il momento storico nei suoi giudici. Antonio Carratta ricorda che agli inizi del secolo scorso Giuseppe Chiovenda collocava propriamente il dilemma della legislazione processuale tra predeterminazione e discrezionalità:

(...)se le forme devono essere prestabilite dalla legge o se debba essere lasciato all'arbitro del giudice di regolarle volta per volta, secondo le esigenze del caso concreto", ribadendo che l'amplificazione dei poteri direttivi del giudice è "un mezzo potente di semplificazione processuale ${ }^{68}$.

Proprio a tale riguardo, la Corte costituzionale italiana ha riconosciuto che $\mathrm{i}$ principi costituzionali che fondano il giusto processo non richiedono l'esistenza di un unico modello di rito processuale ${ }^{69}$; vale a dire che il legislatore può disporre del processo con una certa libertà.

La stessa Corte costituzionale ha affermato, in altro momento, che la durata del processo, per essere ragionevole, nei termini del $2^{\circ}$ comma dell'art. 111 Cost., deve

\footnotetext{
65 "La storia del diritto processuale insegna che la semplificazione e deformalizzazione del giudizio, anche ove concerna la fase di assunzione delle prove, non comporta di per sé l'impossibilità di un accertamento completo dei fatti. Ricorda non a caso Chiovenda che l'essere la cognizione completa o non, riguarda le condizioni del provvedimento del giudice e non le forme del processo, e che la cognizione è completa quando il convenuto è ammesso a far valere tutte le eccezioni contro la domanda e quando la domanda è esaminata in tutti i suoi elementi costitutivi, il che può avvenire in forme più complesse o più semplici" (CHIOVENDA G., Istituzioni di diritto processuali civili I, p. 216).

${ }^{66}$ CARRATTA A., Struttura e funzione nei procedimenti giurisdizionali sommari, in La tutela Sommaria in Europa-Studi, (a cura di) Antonio Carratta, Jovene Editore 2012, p. 1 a 34.

${ }^{67}$ IVI, p. 3

${ }^{68}$ CARRATTA A., Struttura e funzione, cit., p. 2.

${ }^{69}$ Corte cost., 14 dicembre 1989, n. 543, in Foro it., 1990, I, 366 ss., con osservazioni di Proto Pisani menzionata dal Carratta in IVI, p. 20.
} 
Revista Eletrônica de Direito Processual - REDP.

Rio de Janeiro. Ano 12. Volume 19. Número 2. Maio a Agosto de 2018

Periódico Quadrimestral da Pós-Graduação Stricto Sensu em Direito Processual da UERJ

Patrono: José Carlos Barbosa Moreira (in mem.). ISSN 1982-7636. pp. 84-117

www.redp.uerj.br

"conciliarsi anche con le altre tutele costituzionali e con il diritto delle parti di agire e difendersi in giudizio garantito dall'art. 24 Cost." ${ }^{\text {70. }}$

Merita di essere evidenziato che "la deviazione dalle forme del processo a cognizione piena ed esauriente perché possa determinare la sommarietà va necessariamente valutata alla luce della sua incidenza sul risultato finale della cognizione del giudice ${ }^{71}$ ". Questo risultato finale dipende dal grado di semplificazione, in modo che laddove essa non garantisca alle parti l'esercizio di poteri processuali nucleari alla cognizione piena e esauriente, comporta non soltanto una semplificazione ma anche una sommarietà della cognizione. Non potendo in questa sede ripercorrere del tutto il dibattito sul tema della semplificazione delle forme processuali, sommarietà della cognizione e procedimenti sommari a cognizione piena, i quali esigono una discussione più approfondita, ci limitiamo a indicare brevemente questi superficiali spunti.

\section{Case management nell'ordinamento italiano}

Il Codice di Procedura Civile italiano del 1942 è stato sottoposto a riforme importanti che lo hanno aggiornato ed hanno modificato in maniera significante la sua struttura. Un esempio è la riforma dalla Legge 533/1973, Legge del Lavoro, con la quale è stato introdotto il rito processuale del lavoro, con un conseguente cambiamento completo del rito ordinario. Inoltre, negli ultimi trent'anni il Codice di Procedura Civile è stato modificato molte volte ed in modo più ampio, negli anni '90 e '95.

La riforma del 1990 aveva un approccio favor actoris e aveva come traguardo quello di accelerare la procedura, dando maggiore effettività alla tutela dei diritti, nonché quella di scoraggiare l'abuso del diritto di difesa. Nonostante questi sforzi, la riforma successiva del 1995, introdotta con la Legge 534/1995, ha permesso l'immissione di nuovi elementi di prova, cercando di riequilibrare i poteri tra le parti.

La riforma italiana del 2005 (con la 1. 14 maggio 2005, n. 80: c.d. legge competitività) ha poi abbandonato la nozione di cooperazione tra le parti, in contrasto con quella che è diventata la nuova faccia del processo inglese. Le nuove regole avevano come

\footnotetext{
${ }^{70}$ Corte cost., 9 febbraio 2001, n. 32, in Giust. Civ., 2001, I, 1171ss.

${ }^{71}$ IVI, p. 27.
} 
Revista Eletrônica de Direito Processual - REDP.

Rio de Janeiro. Ano 12. Volume 19. Número 2. Maio a Agosto de 2018

Periódico Quadrimestral da Pós-Graduação Stricto Sensu em Direito Processual da UERJ

Patrono: José Carlos Barbosa Moreira (in mem.). ISSN 1982-7636. pp. 84-117

www.redp.uerj.br

obiettivo quello di dare ai convenuti più tempo per "sistemare" i casi e per modificare le sue difese.

L'articolo 81-bis delle Disposizioni di attuazione del Codice di Procedura Civile (Disp. Att. c.p.c), introdotto dalla legge 69/2009, e l'art. 175 c.p.c. stabiliscono che il giudice può fissare un calendario del processo all'inizio della domanda. Questa disposizione è ispirata alla disciplina francese del calendrier du procès. Nel procedimento inglese, d'altra parte, ci sono disposizioni simili che parlano di trial date o trial "window", essendo poteri delle parti e del giudice quelli di fissare il tempo del processo.

Tuttavia, la procedura civile italiana è ancora priva di un sistema sanzionatorio che garantisca l'osservanza del calendario processuale; di conseguenza, l'apertura del sistema non trasferisce coercibilità ed efficienza al processo. Il sistema inglese, invece, conta su un sistema coercitivo strutturato con forti sanzioni ${ }^{72}$.

È un codice, pertanto, con un carattere molto specifico e basato su punti riguardo ai quali non sono state date, in tutti questi anni, molte concessioni. Per fare un esempio, l'oralità è sempre perseguita attraverso le udienze di trattazione, che risultano comunque sempre presenti; o ancora il rito d'appello, che porta con sé un'importante fasciatura garantista, così come tanti altri elementi, che lasciano trasparire nell'intento del legislatore una mancanza di fiducia nel giudice abbastanza palese.

Oltre a ciò, spesso si afferma che il codice originario desiderava dare al giudice un ruolo più attivo, ma questa è in verità una bozza di una realtà che non si è mai realizzata $^{73}$.

Si parla, inoltre, moltissimo di poteri di direzione e controllo del processo: questi ultimi possono effettivamente condurre ad un processo più corretto, democratico e, per

\footnotetext{
${ }^{72}$ BONCI, op. cit., p. 231: "What is more, the Italian judge lacks effective sanctions if a party breaches the timelimits contained in the order. In England the judges will fix the timetable for each case on the multi-track (covering cases of high financial significance) in consultation with the parties and their lawyers. To support this system, the English courts have a range of strong sanctions, notably cost orders, the power to stay proceedings, the making of "unless orders" which provide for automatic striking out of claims or defences where there has been breach of such a "peremptory" procedural order made by the court (although the court can award relief against such an automatic sanction, if the penitent party convinces the court that this is fair and reasonable, in the light of various criteria listed in the English procedural codes".

${ }_{73}^{73}$ FICCARELLI B., Fase preparatoria del processo civile e case management giudiziale, Napoli: Edizioni Scientifiche Italiane, 2011, p. 15: "E ai poteri del giudice istruttore è significativamente intitolata la Sezione I del Capo II del titolo I del libro II del codice di rito, anche se in pratica l'enunciazione è rimasta allo stadio della dichiarazione di intenti e le riforme processuali che si sono succedute nel tempo non hanno apportato modifiche tali da mettere il giudice in condizione di esercitare effettivamente tutti gli ampi poteri di cui dispone".
} 
Revista Eletrônica de Direito Processual - REDP.

Rio de Janeiro. Ano 12. Volume 19. Número 2. Maio a Agosto de 2018

Periódico Quadrimestral da Pós-Graduação Stricto Sensu em Direito Processual da UERJ

Patrono: José Carlos Barbosa Moreira (in mem.). ISSN 1982-7636. pp. 84-117

www.redp.uerj.br

quanto possibile, celere. Questi poteri non oscurerebbero il principio dispositivo, ma si

limiterebbero ad aspetti della cosa allegata in giudizio, cioè, nell'ambito dell'oggetto

materiale del processo, il quale rimane comunque nel monopolio delle parti.

La direzione del processo è divisa in "direzione materiale" e "direzione

formale": la prima riguarda le attribuzioni e le iniziative ufficiali del giudice nella trattazione o nell'istruzione della causa; la seconda riguarda la conduzione strumentale, le udienze e le attività regolamentari, poteri di impulso formale e meccanico.

Sono classificati come poteri di direzione formale ${ }^{74}$ : "la disposizione di cui all'art. 187 c.p.c., comma 5 ("Il giudice dà ogni altra disposizione relativa al processo")", e anche:

(...) i poteri con cui il giudice rileva le nullità degli atti introduttivi e ne dispone la rinnovazione; adotta i provvedimenti opportuni per la regolarizzazione degli atti "difettosi" o per l'eliminazione di un difetto di rappresentanza, di assistenza o di autorizzazione delle parti, statuisce in merito alla mancata comparizione delle parti, ripristina l'osservanza delle disposizioni sull'assegnazione degli affari alle sezioni ecc. (per es., gli artt. 164 e 291 c.p.c.); i poteri di fissazione delle udienze successive alla prima ed i poteri di fissazione o di proroga dei termini, entro $i$ quali le parti hanno, di volta in volta, l'obbligo o l'onere di compiere gli atti processuali nel loro interesse ${ }^{75}$.

Quanto ai poteri di direzione materiale del procedimento, sono quelli che riguardano il rapporto giuridico processuale o sostanziale:

(...) poteri di iniziativa d'ufficio con i quali il giudice chiarisce $\mathrm{i}$ temi controversi, integra le prove dedotte dalle parti e concorre, quindi, alla formazione del materiale decisorio. Pertanto, nella "direzione materiale", secondo la concezione prevalente, si fanno rientrare: i poteri di autorizzazione con cui il giudice non solo condiziona la chiamata di terzi in giudizio, ma pure incide sulla possibilità di modificazione delle domande, delle eccezioni o delle conclusioni già formulate dalle parti (art. 183, comma 5 c.p.c.; art. 269 c.p.c.; art.

74 CARRATTA A., Funzione sociale e processo civile fra XX e XXI secolo. Disponibile su: http://romatrepress.uniroma3.it/ojs/index.php/funzione/article/view/506/503, accesso il 17 ottobre 2017, p. 122: "Il giudice, nell'ambito di un processo incentrato sul contatto diretto e immediato con le parti, non può assumere una funzione meramente passiva, ma deve avere poteri di direzione della trattazione. E questa fondamentale impostazione relativa al processo civile romano non avrebbe potuto non avere la sua rilevanza anche nella riflessione intorno alla 'romanità' del processo civile moderno, dove proprio in quegli anni si consolida la convinzione che anche nel sistema processuale moderno non sia sufficiente avere un giudice che partecipa solo formalmente allo svolgimento del processo (formelle Prozessleitung), ma che ci sia la necessità, anche al fine di far coincidere la verità processuale con quella materiale, che egli partecipi alla formazione del materiale di causa (dell'«istituto giuridico» oggetto della controversia) mediante la cooperazione con le parti (materielle Prozessleitung), con la conseguente sostituzione dell'Offizialmaxime al Parteidispositionsmaxime".

${ }^{75}$ FICCARELLI B., ult. op. cit., p. 26. 
Revista Eletrônica de Direito Processual - REDP.

Rio de Janeiro. Ano 12. Volume 19. Número 2. Maio a Agosto de 2018

Periódico Quadrimestral da Pós-Graduação Stricto Sensu em Direito Processual da UERJ

Patrono: José Carlos Barbosa Moreira (in mem.). ISSN 1982-7636. pp. 84-117

www.redp.uerj.br

420 c.p.c. comma 1); i poteri ufficiosi di integrazione del contraddittorio (artt. 102 e 107 c.p.c. in relazione all'art. 183, comma 1); i poteri di interrogatorio libero per la chiarificazione dei fatti di causa ed il tentativo di conciliazione (artt. 117; 183 comma 3 e 9; art. 185, comma 1; art. 420 c.p.c, comma 1); la rimessione in termini delle parti; il potere di richiedere alle parti, sulla base dei fatti allegati, i chiarimenti necessari, nonché il parallelo potere di indicare loro le questioni rilevabili d'ufficio che, in varia misura, permettono al giudice di integrare le prove acquisite o assunte per iniziativa delle parti onerate (art. 115, comma 1); il potere di disporre d'ufficio l'ammissione di ogni mezzo di prova, anche al di fuori dei limiti stabiliti dal codice civile e l'assunzione di mezzi informativi specifici (art. 425 c.p.c.), potere attribuito al giudice nelle controversie di lavoro76.

Beatrice Ficcarelli ${ }^{77}$ sostiene che il sistema processuale civile italiano non richiede l'aumento dei poteri del giudice, ma solo che occorrano revisioni alla legge vigente in modo da far convergere quei poteri di direzione, di controllo e gli altri poteri tesi al più sollecito svolgimento del processo, nella direzione di modellare il procedimento con le caratteristiche di ciascuna domanda. Questo approccio darebbe l'elasticità che manca al processo civile italiano.

Nonostante la dottrina italiana abbia lavorato sull'attivismo procedurale giudiziario, la legislazione non ha mai concesso veri poteri pratici di case management.

Questa è la conclusione di Bonci: "the result of this failure by Italian legislature to equip judges with adequate case management powers is that the notorious three-headed monster of costs, complexity and delay continues to bestride the Italian legal system "78.

Altri teorici sostengono, per altro verso, che l'articolo 702-ter del c.p.c. costituisce l'apertura del sistema italiano ad un modello deformalizzato, lo stesso ruolo realizzato dal calendario del processo (art. 81 - ter disp att.), pur non ritenendo che si possa parlare di un vero e proprio caso di case management. D'altro canto, discipline come quella del calendario processuale, vengono ritenute di scarsa applicazione pratica nel sistema italiano. Nonostante le opinioni più scettiche, la disciplina del 702-ter e dell'art. 183 - 2. bis costituiscono, concretamente, la possibilità del giudice di scegliere e stabilire il rito, secondo le necessità della domanda formulata, benché si abbia ancora la necessità che il sistema modifichi e adatti il codice al fine di ottimizzare l'efficienza di tali scelte.

\footnotetext{
${ }^{76}$ IVI, p. 28.

77 IVI, p. 29.

${ }^{78}$ BONCI, op. cit., p. 7.
} 
Revista Eletrônica de Direito Processual - REDP.

Rio de Janeiro. Ano 12. Volume 19. Número 2. Maio a Agosto de 2018

Periódico Quadrimestral da Pós-Graduação Stricto Sensu em Direito Processual da UERJ

Patrono: José Carlos Barbosa Moreira (in mem.). ISSN 1982-7636. pp. 84-117

www.redp.uerj.br

L'articolo 702 bis del c.p.c. italiano disciplina il procedimento sommario di

cognizione, tipo di rito deformalizzato, senza predeterminazione, nel quale il giudice adatta

il procedimento al diritto soggettivo discusso nella domanda senza violare i diritti

fondamentali di difesa delle parti. Il procedimento si apre, quindi, alla discrezionalità del giudice, che lo determina e gestisce ${ }^{79}$ e costituisce una manifestazione italiana dell'elasticità e della proporzionalità nell'ambito della procedura civile ${ }^{80}$.

Sul punto, Luca Passanante sostiene che la possibilità di cambiare il rito da ordinario a sommario "appare tuttavia frutto dell'improvvisazione" $"$, poco utile agli operatori del diritto, in quanto il processo ordinario non è adatto a ospitare una tale decisione nell'ambito dell'udienza dell'art. 183 c.p.c.: “I conditori, inoltre, sembrano non aver tenuto conto del fatto che, nella prassi, il giudizio sommario di cognizione è stato tiepidamente accolto dai giudici, i quali hanno spesso manifestato la tendenza a convertire i riti sommari in ordinari”.

In ogni caso vi è un certo consenso sull'idea che il rito sommario, vincolato a cognizione piena, possa rappresentare la soluzione adeguata al problema della durata dei processi. Se la cognizione piena tradizionale non consente un giudizio efficiente e adeguato alla società moderna, tantomeno implica un aumento della qualità del giudizio. La sommarizzazione, appare quindi, come un campo di ricerca appropriato e di grande contenuto gnoseologico, tale da essere sviluppato. È la nuova/vecchia frontiera della procedura.

Come ha dimostrato il processo civile italiano, anche se ancora in modo riservato, le porte all'adattabilità, elasticità e proporzionalità sono aperte.

\footnotetext{
${ }^{79}$ FICARELLI B., op. cit., p. 192: "Dopo il primo momento di contatto tra le parti ed il giudice, il procedimento è cosi lasciato alla più ampia discrezionalità di quest'ultimo, che diventa vero "governatore assoluto" del processo".

${ }^{80}$ Vedi LUPOI M. A., Sommario (Ma Non Troppo), in Riv. trim. dir. proc. civ., fasc.4, 2010, pag. 1225: "Così facendo, il legislatore si è avvicinato all'esperienza di altri ordinamenti stranieri, in cui da tempo si sono introdotti meccanismi per adattare gli schemi processuali alla minore o maggiore complessità della singola lite. In realtà, però, le più recenti esperienze estere, come quella inglese o francese (3), si sono mosse sul piano della cooperazione e della collaborazione, nella fase introduttiva della lite, tra parti e giudice, inserendo elementi di negozialità nelle dinamiche processuali e configurando, dopo la proposizione della domanda con modalità unitarie, la possibilità di sviluppare l'iter procedimentale secondo itinerari diversi, a seconda delle caratteristiche delle singole fattispecie. Il più timido legislatore italiano, dal canto suo, ha preferito varare un nuovo modello procedimentale con funzione alternativa e non sostitutiva rispetto a quello ordinario".

${ }^{81}$ PASSANANTE L., Brevi note critiche sul d.d.l. delega per l'efficienza del processo civile, in Riv. trim. di dir. e proc. civ., fasc.2, 2014, pag. 819.
} 
Revista Eletrônica de Direito Processual - REDP.

Rio de Janeiro. Ano 12. Volume 19. Número 2. Maio a Agosto de 2018

Periódico Quadrimestral da Pós-Graduação Stricto Sensu em Direito Processual da UERJ

Patrono: José Carlos Barbosa Moreira (in mem.). ISSN 1982-7636. pp. 84-117

www.redp.uerj.br

In questo senso, è stato ritenuto che dare al giudice l'opportunità di fare una

valutazione del rito più adeguata rappresenti un vero e proprio esercizio di case management italiano ${ }^{82}$.

\section{Conclusioni}

Oltre a rappresentare mere conclusioni, trattasi anche di presentare aspetti che richiedono approfondimenti:

1. La tendenza nel diritto processuale moderno è quella della concessione al giudice di poteri di gestione e di organizzazione del processo con delle libertà. Sistemi adversariali (accusatori) puri tendono ad essere figure anacronistiche e storiche.

2. Mentre nel common Law alcune fasi hanno importanza, nei sistemi di civil Law risulta essere assente l'interesse da parte della procedura civile riguardo al periodo che precede l'inizio formale del processo.

3. Il principio della proporzionalità, avvalorato nel rapporto tra disponibilità di risorse complessive di sistema e ogni singolo processo, è un importante criterio di gestione processuale. Tale ottica merita essere valutata sia de iure condendo, sia de iure condito.

4. La fiducia del periodo storico nei propri giudici è un elemento di rilievo che richiede un approccio più scientifico. Non si può non considerare l'importanza del profilo di giudice scelto per capire il DNA del sistema giudiziario. È necessario pertanto conoscere non solo le idiosincrasie della magistratura, ma anche l'aspetto di razionalità che coinvolge la presa delle decisioni di gestione nell'ambito del processo.

5. La fiducia nei giudici non è solo quella legislativa ma è anche parte della struttura giudiziaria di controllo di merito e legittimità. L'esercizio di questi poteri, che consentano uno spettro decisionale ampio, richiede la preparazione delle corti superiori nel senso ideologico; tali corti non possono esercitare il controllo delle decisioni dei giudici di primo grado senza tenere in conto la teleologia del case management, con il rischio di costringere i giudici ad un approccio riservato e reticente.

6. Le nozioni di rito adeguato e di economia processuale devono prendere in considerazione la totalità delle risorse del sistema, la globalità del carico di lavoro. In tal

\footnotetext{
${ }^{82}$ FICCARELLI B., op. cit., p. 34.
} 
Revista Eletrônica de Direito Processual - REDP.

Rio de Janeiro. Ano 12. Volume 19. Número 2. Maio a Agosto de 2018

Periódico Quadrimestral da Pós-Graduação Stricto Sensu em Direito Processual da UERJ

Patrono: José Carlos Barbosa Moreira (in mem.). ISSN 1982-7636. pp. 84-117

www.redp.uerj.br

senso, il diritto di difesa deve essere osservato e garantito in modo da conservare integro il proprio nucleo.

7. In ultimo, deve essere oggetto d'analisi anche la razionalità del giudice nell'aspetto collettivo, vale a dire come amministratore dell'unità giudiziaria ove esercita il suo potere. Il giudice non ragiona soltanto riguardo al processo individualmente considerato, ma anche sul collettivo di processi e tale razionalità influenza il destino delle domande, benché non vogliamo ammetterlo. Il ruolo del fattore umano: il "fattore umano"e le implicazioni sul successo o fallimento delle innovazioni legislative.

\section{REFERÊNCIAS BIBLIOGRÁFICAS:}

ANDREWS, Neil. Controversie Collettive, Transazione e Conciliazione In Inghilterra. Revista de Processo, vol. 169/2009, p. 62 - 92, Mar / 2009 DTR\2009\197.

ANDREWS, Neil. Fundamental Principles of Civil Procedure: Order Out of Chaos.

Chapter in: Civil Litigation in a Globalising World. Date: 31 January 2012.

ANDREWS, Neil. The Three Paths of Justice. Dordrecht: Springer, 2012.

ANSANELLI, Vincenzo. Flessibilità, proporzionalità ed efficienza. Il nuovo art. 183-bis c.p.c., in Rivista Trimestrale di Diritto e Procedura Civile, fasc.1, 2015.

BIAVATI, Paolo. Osservazioni Sulla Ragionevole Durata Del Processo Di Cognizione, in Rivista Trimestrale di Diritto e Procedura Civile, fasc.2, 2012.

BIAVATI, Paolo. Appunti sulla Struttura della Decisione e L'ordine delle questioni. Rivista Trimestrale di Diritto e Procedura Civile, fasc.4, 2009.

BONCI, Marco. Active Case Management - English reception and italian rejection. Revista de Processo, vol. 219/2013.

CAPONI, Remo. The Performance of the Italian Civil Justice System: An Empirical Assessment. The Italian Law Journal, Vol. 2 - No. 01 (2016).

CAPONI, Remo. Modelli europei del processo di cognizione: l'esempio tedesco, in Questione Giustizia, n. 1, 2006.

CARRATTA, Antonio. Funzione sociale e processo civile fra XX e XXI secolo. Disponibile in: http://romatrepress.uniroma3.it/ojs/index.php/funzione/article/view/506/503. Accesso in: 17 ottobre 2017. 
Revista Eletrônica de Direito Processual - REDP.

Rio de Janeiro. Ano 12. Volume 19. Número 2. Maio a Agosto de 2018

Periódico Quadrimestral da Pós-Graduação Stricto Sensu em Direito Processual da UERJ

Patrono: José Carlos Barbosa Moreira (in mem.). ISSN 1982-7636. pp. 84-117

www.redp.uerj.br

CARRATTA, Antonio. Struttura e funzione nei procedimenti giurisdizionali sommari. In:

La tutela Sommaria in Europa- Studi, (a cura di) Antonio Carratta, Jovene Editore 2012

CARRATTA, Antonio. La "semplificazione" dei riti e le nuove modifiche del processo civile, Torino: Giappichelli Editore, 2012.

CIPRIANI, F. Il processo civile tra vecchie ideologie e nuovi slogan, in Riv. Dir. proc., 2003.

COMOGLIO, Luigi Paolo. Giustizia (Non) A Tutti I Costi. Significativo «Update» delle civil procedure rules inglesi e suggestioni sistematiche per la riforma del processo civile, in Rivista Trimestrale di Diritto e Procedura Civile, fasc.1, 2014, p. 145.

COSTABILE, Felice. Storicità del Diritto Romano e Creatività Dogmatica per il Diritto Moderno. In: Atena e Roma alle origini della democrazia moderna, (a cura di) Felice Costabile. Torino: Giappichelli Editore.

CRISCUOLI, Giovanni e SERIO, Mario. Nuova Indroduzione allo studio del Diritto Inglese - Le Fonti. Milano: Giuffrè Editore, 2016.

DONDI, Angelo- ANSANELLI, Vicenzo- COMOGLIO, Paolo. Processi civili in evoluzione, Milano: Giuffrè Editore, 2015.

DONDI, Angelo. Taruffo Comparatista - Uno Sguardo Originale Ai Problemi Del Processo Civile, in Rivista Trimestrale di Diritto e Procedura Civile, fasc. 2, 2015.

DONZELLI, Romolo. La fase preliminare del nuovo processo civile inglese e l'attività di "case management" giudiciziale, in Davanti al giudice. Studi sul processo societario, (a cura di) L. Lanfranchi e A. Carratta, 2005.

EAGLES, Ian. Disclosure from material obtained from discovery. Disponibile su: http://onlinelibrary.wiley.com/advanced/search/results?searchRowCriteria\%5B0\%5D.fi eldName $=$ author $\&$ start $=1 \&$ resultsPerPage $=20 \&$ searchRowCriteria\%5B0\%5D.queryStr ing=\%22Ian\%20Eagles\%22, accesso il 17 ottobre 2017.

FICCARELLI, Beatrice. Fase preparatoria del processo civile e case management giudiziale. Napoli: Edizione Scientifiche Italiane, 2011.

GARAPON, Antoine. Lo Stato minimo. Il neoliberalismo e la giustizia. Milano: Raffaello Cortone Editore, 2012.

GIORGIANTONIO, Cristina. Le riforme del processo civile italiano tra adversarial system $e$ case management. Disponibile su: http://www.sideisle.it/ocs/viewpaper.php?id=69\&cf=1. Accesso il 17 ottobre 2017. 
Revista Eletrônica de Direito Processual - REDP.

Rio de Janeiro. Ano 12. Volume 19. Número 2. Maio a Agosto de 2018

Periódico Quadrimestral da Pós-Graduação Stricto Sensu em Direito Processual da UERJ

Patrono: José Carlos Barbosa Moreira (in mem.). ISSN 1982-7636. pp. 84-117

www.redp.uerj.br

LANGBEIN, John H.. The German Advantage in Civil Procedure. The University of

Chicago Law Review. Volume 52, number 4, Fall 1985. Disponibile su: http://digitalcommons.law.yale.edu/cgi/viewcontent.cgi?article=1530\&context=fss_pap $\underline{\text { ers, }}$ accesso il 17 ottobre 2017.

LUPOI, Michele Angelo. Sommario (Ma Non Troppo) in Rivista Trimestrale di Diritto e Procedura Civile, fasc.4, 2010.

MANDRIOLI, Crisanto e CARRATTA, Antonio. Diritto Processuale Civile I. Nozioni Introduttive e Disposizioni Generali. Torino: Giappichelli Editore, 24ª edizione, 2015.

MARINARI, Marcello. La riforma Jackson e la disciplina delle spese nel processo civile inglese. Disponibile su: http://www.questionegiustizia.it/articolo/la-riforma-jackson-ela-disciplina-delle-spese-nel-processo-civile-inglese_31-05-2014.php, acesso il 17 ottobre 2017.

PORRECA, Paolo. Il procedimento sommario di cognizione: un rito flessibile, Rivista Trimestrale di Diritto e Procedura Civile, fasc.3, 2010, p. 823.

PASSANANTE, Luca. Brevi note critiche sul d.d.l. delega per l'efficienza del processo civile. Rivista Trimestrale di Diritto e Procedura Civile, fasc.2, 2014.

TARUFFO, Michele. Il processo civile di civil Law e di common Law: aspetti fondamentali, in Sui Confini. Scritti sulla giustizia civile. Bologna: Società Editrice il Mulino, 2002.

ZUCKERMAN, A. A. S. Lord Woolf's Access to Justice: Plus Ça Change, in (1996) 59 Modern Law $77 . \quad$ Review su: http://adrianzuckerman.co.uk/files/File/woolfmlr-jen.dr2.pdf, accesso il 17 ottobre 2017. 